\title{
SPECIALIZATION AND INTEGRAL CLOSURE
}

\author{
JOOYOUN HONG AND BERND ULRICH
}

\begin{abstract}
We prove that the integral closedness of any ideal of height at least two is compatible with specialization by a generic element. This opens the possibility for proofs using induction on the height of an ideal. Also, with additional assumptions, we show that an element is integral over a module if it is so modulo a generic element of the module. This turns questions about integral closures of modules into problems about integral closures of ideals, by means of a construction known as Bourbaki ideal.
\end{abstract}

In this paper we prove in a rather general setting that the integral closure of ideals and modules is preserved under specialization modulo generic elements. Recall that the integral closure $\bar{I}$ of an ideal $I$ in a commutative ring $R$ is the set of all elements $y$ that are integral over $I$, i.e., satisfy a polynomial equation of the form

$$
y^{m}+\alpha_{1} y^{m-1}+\cdots+\alpha_{i} y^{m-i}+\cdots+\alpha_{m}=0,
$$

where $\alpha_{i} \in I^{i}$. Alternatively, one can consider the Rees algebra $\mathcal{R}(I)$ of $I$, which is the subalgebra $R[I t]$ of the polynomial ring $R[t]$. The integral closure $\overline{\mathcal{R}(I)}$ of $\mathcal{R}(I)$ in $R[t]$ is again a graded algebra, and its graded components recover the integral closures of all powers of $I$,

$$
\overline{\mathcal{R}(I)}=R \oplus \bar{I} t \oplus \overline{I^{2}} t^{2} \oplus \cdots \oplus \overline{I^{i}} t^{i} \oplus \cdots .
$$

In our main result, Theorem 2.1, we consider a Noetherian ring $R$ such that the completion $\left(R_{\mathfrak{m}} / \sqrt{0}\right)^{\wedge}$ is reduced and equidimensional for every maximal ideal $\mathfrak{m}$ of $R$; for instance, $R$ could be an equidimensional excellent local ring. For any $R$-ideal $I=\left(a_{1}, \ldots, a_{n}\right)$ of height at least 2 , we prove that

$$
\overline{I^{\prime} /(x)}=\overline{I^{\prime}} /(x),
$$

where $x=\sum_{i=1}^{n} z_{i} a_{i}$ is a generic element for $I$ defined over the polynomial ring $R^{\prime}=$ $R\left[z_{1}, \ldots, z_{n}\right]$ and $I^{\prime}$ denotes the extension of $I$ to $R^{\prime}$. This result can be paraphrased by saying that an element is integral over $I$ if it is so modulo a generic element of the ideal. Other, essentially unrelated, results about lifting integral dependence have been proved by Teissier, Gaffney, and Gaffney and Kleiman ([6], [7], [25], [26]). They go by the name 'Principle of Specialization of Integral Dependence' and play an important role in equisingularity theory.

Our main result, Theorem 2.1, opens the possibility for proofs using induction on the height. This yields, for instance, a quick proof of Huneke's and Itoh's celebrated result on integral closures of powers of complete intersections ([11], [15]): If $R$ is a ring as above and $I$ an $R-$ ideal generated by a regular sequence, then $\overline{I^{n+1}} \bigcap I^{n}=\bar{I} I^{n}$ for every $n \geq 0$ (see Theorem 2.4).

The proof of Theorem 2.1 is based on a vanishing theorem for local cohomology modules; this is natural as the obstruction to the specialization of integral closure lies in a cohomology

AMS 2010 Mathematics Subject Classification. Primary 13B22; Secondary 13B21, 13A30.

Key Words and Phrases. Integral closure, integral dependence, Rees algebra, symmetric algebra.

The first author is partially supported by the Sabbatical Leave Program at Southern Connecticut State University (Spring 2014) and the second author is partially supported by the NSF. 
module. Thus in Theorem 1.2 we consider a ring $R$ as above, a proper $R$-ideal $I$ of positive height, the extended Rees algebra $\mathcal{A}=R\left[I t, t^{-1}\right]$, its integral closure $\overline{\mathcal{A}}$ in $R\left[t, t^{-1}\right]$, and an $\mathcal{A}$-ideal $J$ of height at least 3 generated by $t^{-1}$ and homogeneous elements of positive degree; in this setup we show that the second local cohomology module $H_{J}^{2}(\overline{\mathcal{A}})$ vanishes in non-positive degrees. Theorem 2.1 about the specialization of $\bar{I}$ follows from the vanishing of this module in degree zero; indeed, the algebra $\overline{\mathcal{A}}$ recovers the integral closures of the powers of $I$ as it coincides with $\overline{\mathcal{R}(I)}$ in non-negative degrees. The main idea in the proof of Theorem 1.2 is to pass to the graded ring associated to the filtration of fractional powers $\left\{\left(\overline{I^{n}}\right)^{\frac{1}{e}} \mid n \geq 0\right\}$ and to observe that this ring is reduced for suitable $e$ (Lemma 1.1(b)). One can then use the fact that the first local cohomology module of a non-negatively graded reduced ring vanishes in negative degrees, which translates into the vanishing of the second local cohomology of $\overline{\mathcal{A}}$ in non-positive degrees. Theorem 1.2 and its proof essentially go back to Itoh ([15, Theorem 2 and Lemma 5]), but special care must be exercised due to the fact that the various integral closures appearing in the proof need not be Noetherian. Itoh uses this result to prove his theorem about integral closures of powers of complete intersections ([15, Theorem 1]), described above as Theorem 2.4 his paper [15] is devoted to that proof. Based on this theorem, he gives a proof of specialization of integral closures in [16. Theorem 1], but only for parameter ideals in analytically unramified local Cohen-Macaulay rings; thus our main theorem, Theorem 2.1, was known to Itoh for the class of complete intersection ideals.

Another set of applications of Theorem 2.1 concerns integral closures of modules. Let $R$ be a Noetherian ring and $E$ a finitely generated $R$-module having a rank, say $e$, by which we mean that $K \otimes_{R} E$ is a free module of rank $e$ over the total ring of quotients $K$ of $R$. In this case the Rees algebra $\mathcal{R}(E)$ is defined as the symmetric algebra $\mathcal{S}(E)$ modulo $R$-torsion. The Rees algebra is a standard graded $R$-algebra whose $n^{\text {th }}$ graded component we denote by $E^{n}$. If $E$ is torsion-free, one can embed $E$ into a free module $F$ with basis $\left\{t_{1}, \ldots, t_{m}\right\}$, and we obtain $\mathcal{R}(E)$ as the image of the natural map $\mathcal{S}(E) \rightarrow \mathcal{S}(F)$, or in other words, as the $R$-subalgebra $R[E]$ of the polynomial ring $\mathcal{S}(F)=R\left[t_{1}, \ldots, t_{m}\right]$. In particular, for $E=I \subset R$ an ideal, this notion of Rees algebra coincides with the one given at the beginning of the introduction. If on the other hand $E$ fails to have a rank, then the definition needs to be modified considerably, see [5].

Once the notion of Rees algebra is in place, one can easily introduce the concepts of reduction, integral dependence, and integral closure of modules. Given two submodules $U \subset V$ of $E$, we say that $V$ is integral over $U$ in $E$ if the inclusion of subalgebras of $\mathcal{R}(E)$,

$$
R[U] \subset R[V]
$$

is an integral ring extension. The module $E$ is integral over $U$, or $U$ is a reduction of $E$, if $E$ is integral over $U$ in $E$, or equivalently, $E^{n+1}=U E^{n}$ for some $n=n_{0}$ and hence every $n \geq n_{0}$. Finally, the integral closure $\bar{U}^{E}$ of $U$ in $E$ is the unique largest submodule of $E$ that contains $U$ and is integral over $U$ in $E$; it can also be described as the degree one component of the integral closure of the subring $R[U]$ in $\mathcal{R}(E)$. If $\bar{U}^{E}=U$ we say that $U$ is integrally closed in $E$. Now let $R$ be a Noetherian normal ring and $U$ a finitely generated torsion-free $R$-module having a rank $e$. We can embed $U$ into a free module $F=R^{e}$ of the same rank $e$. As $\mathcal{R}(U) \hookrightarrow \mathcal{R}(F)$ is a birational extension and $\mathcal{R}(F)=\mathcal{S}(F)$ is a normal ring, we see that the integral closure $\overline{\mathcal{R}(U)}$ of $\mathcal{R}(U)$ in $\mathcal{R}(F)$ coincides with the integral closure of $\mathcal{R}(U)$ in its total ring of quotients, and hence does not depend on the embedding $U \hookrightarrow F$ of $U$. Thus we call $\bar{U}^{F}$ the integral closure of $U$ and write $\bar{U}=\bar{U}^{F}$. We say that $U$ is integrally closed 
provided $\bar{U}=U$. As it turns out, $\overline{U^{n}}$ coincides with the degree $n$ component of $\overline{\mathcal{R}(U)}$ for every $n$ ([22, (A), p.434]), and thus $U$ is said to be normal if $U^{n}$ is integrally closed for every $n$, or equivalently, if $\mathcal{R}(U)$ is a normal ring.

To state our main theorem about specialization of integral closures of modules, Theorem 4.4 . let $R$ be a Noetherian normal ring such that for every maximal ideal $\mathfrak{m}$ of $R$ the completion $\widehat{R_{\mathfrak{m}}}$ is reduced and equidimensional, and let $E=\sum_{i=1}^{n} R a_{i}$ be a torsion-free $R$-module of rank $e \geq 2$ such that for every prime ideal $\mathfrak{p}$ of $R$ with depth $R_{\mathfrak{p}} \leq 2$ either $E_{\mathfrak{p}}$ has a nontrivial free direct summand or $R_{\mathfrak{p}}$ is regular. Under these hypotheses we prove that

$$
\overline{E^{\prime} / R^{\prime} x}=\overline{E^{\prime}} / R^{\prime} x,
$$

where $x=\sum_{i=1}^{n} z_{i} a_{i}$ is a generic element for $E$ defined over the polynomial ring $R^{\prime}=$ $R\left[z_{1}, \ldots, z_{n}\right]$ and $E^{\prime}=R^{\prime} \otimes_{R} E$. The significance of this theorem is that it turns questions about integral closures of modules into problems about integral closures of ideals, by means of a construction known as Bourbaki ideal. In fact, factoring out the submodule generated by $e-1$ generic elements $x_{1}, \ldots, x_{e-1}$ for $E$, one obtains an ideal $I=I(E)$ called the generic Bourbaki ideal of $E$. Here one assumes $(R, \mathfrak{m})$ is local and one works in the localized polynomial $\operatorname{ring} R^{\prime \prime}=R\left[\left\{z_{i j}\right\}\right]_{\mathfrak{m} R\left[\left\{z_{i j}\right\}\right]}$ and the module $E^{\prime \prime}=R^{\prime \prime} \otimes_{R} E$. As an immediate consequence of Theorem 4.4 we deduce that

$$
\bar{I}=\overline{E^{\prime \prime}} / \sum_{i=1}^{e-1} R^{\prime \prime} x_{i},
$$

provided $R$ is a ring as in the theorem and $E$ is a finitely generated torsion-free $R$-module of rank $e>0$ such that for every prime ideal $\mathfrak{p}$ of $R$ with depth $R_{\mathfrak{p}} \leq 2$ either $E_{\mathfrak{p}}$ has a free direct summand of rank $e-1$ or $R_{\mathfrak{p}}$ is regular, see Corollary 4.5.

Using this approach, one immediately deduces the results of Kodiyalam and of Katz and Kodiyalam ([17], [18]) about integrally closed modules over two-dimensional regular local rings from the corresponding classical theory for ideals, going back to Zariski ([12], [27]), see Corollary 3.8. The proof of Theorem 4.4 requires an adaptation of Theorem 2.1 to modules, see Theorem 4.3, as well as a separate treatment of the case of two-dimensional regular local rings, see Theorem 3.6. To show the latter result we use aspects of Kodiyalam's work, which we summarize in Theorem 3.2, For the most part however, we recover his results through the method of Bourbaki ideals.

\section{A VANishing THEOREM FOR LOCAL COHOMOLOGY MODUles}

In this section we present the vanishing theorem that will be used in the proof of our main result about specialization of integral closure.

Let $R$ be a Noetherian ring and $I$ an $R$-ideal. Denote the extended Rees ring of $I$ by $\mathcal{A}=R\left[I t, t^{-1}\right]$ and the integral closure of $\mathcal{A}$ in $R\left[t, t^{-1}\right]$ by $\overline{\mathcal{A}}$. Fix an integer $e>0$. Let $u$ be a variable with $u^{e}=t$ and $\operatorname{deg} u=\frac{1}{e}$. Thus the $R$-algebra $R\left[u, u^{-1}\right]$ is graded by $\frac{1}{e} \mathbb{Z}$ and it contains $R\left[t, t^{-1}\right]$ as a Veronese subring. Consider the finitely generated graded $R$-subalgebra $\mathcal{S}=\mathcal{A}\left[u^{-1}\right]$ of $R\left[u, u^{-1}\right]$. This ring is of the form

$$
\mathcal{S}=\cdots \oplus R u^{-1} \oplus R \oplus I u \oplus I u^{2} \oplus \cdots \oplus I u^{e} \oplus I^{2} u^{e+1} \oplus I^{2} u^{e+2} \oplus \cdots \oplus I^{2} u^{2 e} \oplus \cdots .
$$

Notice that $\mathcal{A}$ is a Veronese subring of $\mathcal{S}$. Denote the integral closure of $\mathcal{S}$ in $R\left[u, u^{-1}\right]$ by $\overline{\mathcal{S}}$. The ring $\overline{\mathcal{S}}$ is a graded $R$-subalgebra of $R\left[u, u^{-1}\right]$ and $\overline{\mathcal{A}}$ is a Veronese subring of $\overline{\mathcal{S}}([24$, 2.3.2]). Note that $\overline{\mathcal{S}}$ and $\overline{\mathcal{A}}$ need not be Noetherian though. Let $a$ be an element of $R$ and $n$ 
an integer. Then $a u^{n} \in \overline{\mathcal{S}}$ if and only if $a^{e} t^{n} \in \overline{\mathcal{A}}$, or equivalently, $a^{e} \in \overline{I^{n}}$, where $I^{n}=R$ if $n \leq 0$. Write $\left(\overline{I^{n}}\right)^{\frac{1}{e}}=\left\{a \in R \mid a^{e} \in \overline{I^{n}}\right\}$. Thus $\overline{\mathcal{S}}$ is of the form

$$
\overline{\mathcal{S}}=\bigoplus_{n \in \mathbb{Z}}\left(\overline{I^{n}}\right)^{\frac{1}{e}} u^{n}
$$

Finally, for a ring $R$ with nilradical $\sqrt{0}$, we denote $R / \sqrt{0}$ by $R_{\text {red }}$. We say that a Noetherian local ring is analytically unramified if its completion $\widehat{R}$ is reduced and that a Noetherian ring is locally analytically unramified if each of its localizations at a maximal ideal is analytically unramified.

In the next lemma we prove that for a suitable choice of $e$, the associated graded ring

$$
\mathcal{E}=\overline{\mathcal{S}} / u^{-1} \overline{\mathcal{S}}=\bigoplus_{n \in \mathbb{Z}_{\geq 0}}\left(\left(\overline{I^{n}}\right)^{\frac{1}{e}} /\left(\overline{I^{n+1}}\right)^{\frac{1}{e}}\right)
$$

is reduced. From this we deduce that $\mathcal{E}$ is Noetherian and that its integral closure in the total ring of quotients is non-negatively graded, though not necessarily Noetherian.

Lemma 1.1. Let $R$ be a Noetherian, equidimensional, universally catenary local ring of dimension $d$ such that $R_{\mathrm{red}}$ is analytically unramified. Let $I=\left(a_{1}, \ldots, a_{n}\right)$ be a proper $R$-ideal with $\operatorname{ht}(I)>0$ and write $\mathcal{A}=R\left[I t, t^{-1}\right]$ for the extended Rees ring of $I$. Let $v_{1}, \ldots, v_{r}$ be the Rees valuations of $I$ ([24, 10.1.1]), and let $e$ be the least common multiple of the values $v_{1}(I), \ldots, v_{r}(I)$ of $I$. Let $u$ be a variable with $u^{e}=t$ and $\operatorname{deg} u=\frac{1}{e}$. Write $\mathcal{S}=\mathcal{A}\left[u^{-1}\right]$ and let $\overline{\mathcal{S}}$ be the integral closure of $\mathcal{S}$ in $R\left[u, u^{-1}\right]$.

(a) Let $\overline{\mathcal{S}_{\text {red }}}$ denote the integral closure of $\mathcal{S}_{\text {red }}$ in $R_{\text {red }}\left[u, u^{-1}\right]$. Then the $R$-algebra $\overline{\mathcal{S}_{\text {red }}}$ is finitely generated and graded by $\frac{1}{e} \mathbb{Z}$, has a unique maximal homogeneous ideal, which is a maximal ideal, and is equidimensional of dimension $d+1$.

(b) One has the equality $\overline{\mathcal{S}} / u^{-1} \overline{\mathcal{S}}=\overline{\mathcal{S}_{\text {red }}} / u^{-1} \overline{\mathcal{S}_{\text {red }}}$. This $R$-algebra is finitely generated and graded by $\frac{1}{e} \mathbb{Z}_{\geq 0}$, has a unique maximal homogeneous ideal, is equidimensional of dimension $d$, and is reduced.

(c) Let $R^{\prime}=R\left[z_{1}, \ldots, z_{n}\right]$ be a polynomial ring in the variables $z_{1}, \ldots, z_{n}, I^{\prime}=I R^{\prime}$, and $x=\sum_{i=1}^{n} z_{i} a_{i}$. Then $t^{-1}, x t$ is a regular sequence on $R^{\prime} \otimes_{R} \overline{\mathcal{A}}$. In particular, $x t$ is regular on $R^{\prime} \otimes_{R}\left(\overline{\mathcal{A}} / t^{-1} \overline{\mathcal{A}}\right)$.

(d) Write $\mathcal{E}=\overline{\mathcal{S}} / u^{-1} \overline{\mathcal{S}}$ and let $\overline{\mathcal{E}}$ be the integral closure of $\mathcal{E}$ in its total ring of quotients. Then $\overline{\mathcal{E}}$ is a finite direct product of Krull domains that are graded by $\frac{1}{e} \mathbb{Z}_{\geq 0}$. In particular, $\overline{\mathcal{E}}$ is graded by $\frac{1}{e} \mathbb{Z}_{\geq 0}$.

Proof. (a) Let $I_{\text {red }}$ be the image of $I$ in $R_{\text {red }}$. We denote the degree $m$ component by [ $]_{m}$. Then

$$
\left[\overline{\mathcal{S}_{\text {red }}}\right] \frac{n}{e} \cdot u^{-n}=\left(\overline{I_{\text {red }}^{n}}\right)^{\frac{1}{e}} \subset\left(\overline{I_{\text {red }}^{\left\lfloor\frac{n}{e}\right\rfloor}}\right)^{\frac{1}{e}}=\overline{I_{\text {red }}^{\left\lfloor\frac{n}{e}\right\rfloor}} \subset I_{\text {red }}^{\left\lfloor\frac{n}{e}\right\rfloor-k},
$$

where the last containment holds for some fixed integer $k \geq 0$ and every integer $n$ because $R_{\text {red }}$ is analytically unramified $([21,1.4])$. On the other hand,

$$
I_{\text {red }}^{\left\lfloor\frac{n}{e}\right\rfloor-k}=\left[\mathcal{S}_{\text {red }}\right]_{\left(\left\lfloor\frac{n}{e}\right\rfloor-k\right)} \cdot u^{-\left(\left\lfloor\frac{n}{e}\right\rfloor-k\right) e} \subset\left[\mathcal{S}_{\text {red }}\right]_{\left(\frac{n}{e}-(k+1)\right)} \cdot u^{-n+(k+1) e} .
$$

Therefore $\overline{\mathcal{S}_{\text {red }}} \subset u^{(k+1) e} \mathcal{S}_{\text {red }}$. Since $\mathcal{S}_{\text {red }}$ is a Noetherian ring, it follows that $\overline{\mathcal{S}_{\text {red }}}$ is a finitely generated $\mathcal{S}_{\text {red }}$-module, hence a finitely generated $R_{\text {red }}$-algebra. We already argued that $\overline{\mathcal{S}_{\text {red }}}$ 
is graded by $\frac{1}{e} \mathbb{Z}$. Writing $\mathfrak{m}$ for the maximal ideal of $R$, we have $(\bar{I})^{\frac{1}{e}} \subset \mathfrak{m}$. Therefore $\left(\overline{\mathcal{S}_{\text {red }}}\right)_{<0} \oplus \mathfrak{m} \oplus\left(\overline{\mathcal{S}_{\text {red }}}\right)_{>0}$ is a maximal ideal of $\overline{\mathcal{S}_{\text {red }}}$ and the unique maximal homogeneous ideal.

Since $\overline{\mathcal{S}_{\text {red }}}$ is torsion-free over $\mathcal{A}_{\text {red }}$ and both rings are Noetherian and reduced, for any minimal prime ideal $\mathfrak{Q}$ of $\overline{\mathcal{S}_{\text {red }}}$, the ideal $\mathfrak{q}=\mathfrak{Q} \cap \mathcal{A}_{\text {red }}$ is a minimal prime ideal of $\mathcal{A}_{\text {red }}$. The extension $\mathcal{A}_{\text {red }} / \mathfrak{q} \subset \overline{\mathcal{S}_{\text {red }}} / \mathfrak{Q}$ is integral and the ring $\mathcal{A}_{\text {red }}$ is equidimensional of dimension $d+1$. Therefore

$$
\operatorname{dim}\left(\overline{\mathcal{S}_{\text {red }}} / \mathfrak{Q}\right)=\operatorname{dim}\left(\mathcal{A}_{\text {red }} / \mathfrak{q}\right)=d+1,
$$

from which we deduce that $\overline{\mathcal{S}_{\text {red }}}$ is equidimensional of dimension $d+1$.

(b) We first show that $u^{-1} \overline{\mathcal{S}}$ is a radical ideal. Thus let $a u^{n} \in \overline{\mathcal{S}}$ be a homogeneous element such that $\left(a u^{n}\right)^{m} \in u^{-1} \overline{\mathcal{S}}$ for some positive integer $m$. Then $a^{m e} \in \overline{I^{n m+1}}$. Hence for each $i=1, \ldots, r$, we have $\frac{e}{v_{i}(I)} v_{i}(a) \geq n+\frac{1}{m}$. Since $e / v_{i}(I)$ is an integer for each $i$, we obtain $\frac{e}{v_{i}(I)} v_{i}(a) \geq n+1$, which means $a^{e} \in \overline{I^{n+1}}$. Therefore $a u^{n+1} \in\left(\overline{I^{n+1}}\right)^{\frac{1}{e}} u^{n+1} \subset \overline{\mathcal{S}}$ and hence $a u^{n} \in u^{-1} \overline{\mathcal{S}}$. Thus we have proved that $u^{-1} \overline{\mathcal{S}}$ is a radical ideal.

Therefore $\overline{\mathcal{S}} / u^{-1} \overline{\mathcal{S}}$ is reduced. Since moreover $(\overline{\mathcal{S}})_{\text {red }}=\overline{\mathcal{S}_{\text {red }}}$, we conclude that $\overline{\mathcal{S}} / u^{-1} \overline{\mathcal{S}}=$ $\overline{\mathcal{S}_{\text {red }}} / u^{-1} \overline{\mathcal{S}_{\text {red }}}$. The remaining assertions follow from part (a) because $u^{-1}$ is a homogeneous regular element on $\overline{\mathcal{S}_{\text {red }}}$ and $\overline{\mathcal{S}_{\text {red }}}$ is a catenary ring.

(c) Write $\mathcal{E}=\overline{\mathcal{S}} / u^{-1} \overline{\mathcal{S}}$. From part (b) we obtain

$$
\operatorname{ht}\left(\mathcal{E}_{+}\right)=\operatorname{dim}(\mathcal{E})-\operatorname{dim}\left(\mathcal{E} / \mathcal{E}_{+}\right)=d-\operatorname{dim}(R / I)>0 .
$$

It follows that grade $\mathcal{E}_{+}>0$ because $\mathcal{E}$ is reduced. We claim that $\mathcal{E}_{+} \subset \sqrt{I^{*} \mathcal{E}}$, where $I^{*}$ denotes the image of $I$ in $\mathcal{E}_{1}$. Any homogeneous element in $\mathcal{E}_{+}$is of the form $\alpha^{*} \in \mathcal{E}_{\frac{k}{e}}$, where $\alpha^{*}$ is the image of an element $\alpha \in\left(\overline{I^{k}}\right)^{\frac{1}{e}}$ and $k$ is a positive integer. Then $\alpha^{e} \in \overline{I^{k}}$. For a sufficiently large integer $l$, we obtain $\alpha^{e l} \in \overline{I^{k l}}=I \overline{I^{k l-1}}$. Notice that $\left(\alpha u^{k}\right)^{e l}=\alpha^{e l} u^{e k l} \in \overline{\mathcal{S}}_{k l}, I u^{e} \subset \overline{\mathcal{S}}_{1}$, and $\overline{I^{k l-1}} u^{e(k l-1)} \subset \overline{\mathcal{S}}_{k l-1}$. Hence $\alpha^{* e l} \in I^{*} \mathcal{E}$, which proves the claim. From the claim we obtain grade $I^{*} \mathcal{E}>0$.

Since the leading form $x^{*}$ of $x$ in $R^{\prime} \otimes_{R} \mathcal{E}$ is a generic element for $I^{*} \mathcal{E}$, it then follows that $x^{*}$ is regular on $R^{\prime} \otimes_{R} \mathcal{E}$. Therefore $u^{-1}, x u^{e}$ is a regular sequence on $R^{\prime} \otimes_{R} \overline{\mathcal{S}}$. We conclude that $\left(u^{-1}\right)^{e}=t^{-1}$, xt is a regular sequence on $R^{\prime} \otimes_{R} \overline{\mathcal{A}}$ because $R^{\prime} \otimes_{R} \overline{\mathcal{A}}$ is a direct summand of $R^{\prime} \otimes_{R} \overline{\mathcal{S}}$. In particular, $x t$ is a regular element on $R^{\prime} \otimes_{R}\left(\overline{\mathcal{A}} / t^{-1} \overline{\mathcal{A}}\right)$.

(d) Since the $\operatorname{ring} \mathcal{E}$ is Noetherian, it has only finitely many minimal prime ideals, say $\mathfrak{Q}_{1}, \ldots, \mathfrak{Q}_{s}$. Notice that $\mathfrak{Q}_{1}, \ldots, \mathfrak{Q}_{s}$ are all homogeneous. Let $\overline{\mathcal{E} / \mathfrak{Q}_{i}}$ denote the integral closure of $\mathcal{E} / \mathfrak{Q}_{i}$ in its quotient field for each $i=1, \ldots, s$. Since $\mathcal{E}$ is reduced, the integral closure $\overline{\mathcal{E}}$ equals $\overline{\mathcal{E} / \mathfrak{Q}_{1}} \times \cdots \times \overline{\mathcal{E} / \mathfrak{Q}_{s}}$. By [24, 4.10.5 and 2.3.6], the integral closures $\overline{\mathcal{E} / \mathfrak{Q}_{i}}$ are Krull domains and are graded by $\frac{1}{e} \mathbb{Z}_{\geq 0}$. In particular, $\overline{\mathcal{E}}$ is graded by $\frac{1}{e} \mathbb{Z}_{\geq 0}$.

Here is the vanishing theorem that will be used in the next section.

Theorem 1.2. Let $R$ be a Noetherian, locally equidimensional, universally catenary ring such that $R_{\text {red }}$ is locally analytically unramified. Let $I$ be a proper $R$-ideal with $\operatorname{ht}(I)>0, \mathcal{A}=$ $R\left[I t, t^{-1}\right]$ the extended Rees ring of $I$, and $\overline{\mathcal{A}}$ the integral closure of $\mathcal{A}$ in $R\left[t, t^{-1}\right]$. Let $J$ be an $\mathcal{A}$-ideal of height at least 3 generated by $t^{-1}$ and homogenous elements of positive degree. Then $\left[H_{J}^{2}(\overline{\mathcal{A}})\right]_{n}=0$ for all $n \leq 0$, where []$_{n}$ denotes the degree $n$ component. 
Proof. By localizing, we may assume that $R_{\text {red }}$ is an analytically unramified Noetherian local ring. Let $\mathcal{S}=\mathcal{A}\left[u^{-1}\right]$, where $u$ is a variable with $u^{e}=t$ and $\operatorname{deg} u=\frac{1}{e}$ and $e$ is the least common multiple of the values of $I$ under its Rees valuations. Denote the integral closure of $\mathcal{S}$ in $R\left[u, u^{-1}\right]$ by $\overline{\mathcal{S}}$. It suffices to show that $\left[H_{J}^{2}(\overline{\mathcal{S}})\right]_{n}=0$ for any rational number $n \leq 0$ because $\overline{\mathcal{A}}$ is a Veronese subring of $\overline{\mathcal{S}}$. Let $d=\operatorname{dim} R$. Write $\mathcal{E}=\overline{\mathcal{S}} / u^{-1} \overline{\mathcal{S}}$ and let $\overline{\mathcal{E}}$ be the integral closure of $\mathcal{E}$ in its total ring of quotients. Then $\mathcal{E}$ is a finitely generated $R$-algebra and $\overline{\mathcal{E}}$ is graded by $\frac{1}{e} \mathbb{Z}_{\geq 0}$ (Lemma 1.1(b),(d)). Some of the technical difficulties in the following proof stem from the fact that the ring $\overline{\mathcal{E}}$ may be non-Noetherian.

First we show that $\operatorname{ht}(J \mathcal{F}) \geq 2$ for any finitely generated graded $R$-subalgebra $\mathcal{F}$ of $\overline{\mathcal{E}}$ containing $\mathcal{E}$. Since $\operatorname{dim}(\mathcal{A} / J) \leq d-2$, we have $\operatorname{dim}(\mathcal{F} / J \mathcal{F}) \leq d-2$. Notice that $J \mathcal{F}$ is a homogeneous ideal and that $\mathcal{F}$ is a catenary ring with a unique maximal homogeneous ideal. Thus it remains to show that $\mathcal{F}$ is equidimensional of dimension $d$. Since $\mathcal{F}$ is a torsion-free $\mathcal{E}$-module and $\mathcal{E}$ is Noetherian and reduced (Lemma 1.1(b)), for any minimal prime ideal $\mathfrak{Q}$ of $\mathcal{F}$, the ideal $\mathfrak{q}=\mathfrak{Q} \cap \mathcal{E}$ is a minimal prime of $\mathcal{E}$. The extension $\mathcal{E} / \mathfrak{q} \subset \mathcal{F} / \mathfrak{Q}$ is integral and the ring $\mathcal{E}$ is equidimensional of dimension $d$ (Lemma 1.1(b)). Hence we obtain $\operatorname{dim}(\mathcal{F} / \mathfrak{Q})=\operatorname{dim}(\mathcal{E} / \mathfrak{q})=d$, as asserted.

Next we claim that there exists an $\overline{\mathcal{E}}$-regular sequence of length 2 in $J \overline{\mathcal{E}}$. The integral closure $\overline{\mathcal{E}}$ is a direct product of finitely many Krull domains (Lemma 1.1(d)). Recall that a principal ideal generated by a nonzero element in a Krull domain is a finite intersection of primary ideals of height 1 ([24, 4.10.3]). Hence by Prime Avoidance it suffices to show that $\operatorname{ht}(J \overline{\mathcal{E}}) \geq 2$. Suppose that $h t(J \overline{\mathcal{E}}) \leq 1$. In this case, $J \overline{\mathcal{E}}$ has a minimal prime $\mathfrak{P}$ with $\mathrm{ht}(\mathfrak{P})=\operatorname{ht}(J \overline{\mathcal{E}}) \leq 1$. Since $J \overline{\mathcal{E}}$ is homogeneous, the prime ideal $\mathfrak{P}$ is homogeneous $([3$, 1.5.6(a),(b.i)]). We claim that there exists a homogenous element $y \in J \overline{\mathcal{E}}$ such that $\mathfrak{P}$ is a minimal prime ideal of $y \overline{\mathcal{E}}$. If $\operatorname{ht}(\mathfrak{P})=0$, this is obvious. If $\operatorname{ht}(\mathfrak{P})=\operatorname{ht}(J \overline{\mathcal{E}})=1$, then $J \overline{\mathcal{E}}$ is not contained in any of the finitely many minimal prime ideals of $\overline{\mathcal{E}}$. As $J \overline{\mathcal{E}}$ is generated by homogeneous elements of positive degree, it follows that $J \overline{\mathcal{E}}$ contains a homogeneous element $y$ not contained in any minimal prime of $\overline{\mathcal{E}}([3,1.5 .10])$. Hence $\mathfrak{P}$ is a minimal prime ideal of $y \overline{\mathcal{E}}$ because $\mathrm{ht}(\mathfrak{P})=1$. Since the ideal $J$ is finitely generated, $y \overline{\mathcal{E}}_{\mathfrak{P}}$ contains $J^{n} \overline{\mathcal{E}}_{\mathfrak{P}}$ for some $n$. Let $J^{n}=\left(f_{1}, \ldots, f_{s}\right)$, where $f_{i}$ are homogeneous. For each $i$, there exists a homogeneous element $u_{i} \in \overline{\mathcal{E}} \backslash \mathfrak{P}$ such that $f_{i} u_{i} \in y \overline{\mathcal{E}}$. Write $f_{i} u_{i}=y a_{i}$ for some homogeneous elements $a_{i} \in \overline{\mathcal{E}}$. Let $\mathcal{F}=\mathcal{E}\left[u_{1}, \ldots, u_{s}, a_{1}, \ldots, a_{s}, y\right]$ and set $\mathfrak{p}=\mathfrak{P} \cap \mathcal{F}$. Then $\mathcal{F}$ is a finitely generated graded $R$-subalgebra of $\overline{\mathcal{E}}$ containing $\mathcal{E}$. Hence we have ht $(J \mathcal{F}) \geq 2$ as shown in the preceding paragraph. On the other hand, for every $i, u_{i} \in \mathcal{F} \backslash \mathfrak{p}$ and $y \in \mathfrak{p}$. Since $f_{i} u_{i} \in y \mathcal{F}$, we obtain $f_{i} \in y \mathcal{F}_{\mathfrak{p}}$ for every $i$. Therefore $J^{n} \mathcal{F}_{\mathfrak{p}}$ is contained in $y \mathcal{F}_{\mathfrak{p}}$. But $y \mathcal{F}_{\mathfrak{p}}$ is a proper principal ideal of $\mathcal{F}_{\mathfrak{p}}$ since $y \in \mathfrak{p}$, and the ring $\mathcal{F}_{\mathfrak{p}}$ is Noetherian. Hence $h t(J \mathcal{F}) \leq 1$, which is a contradiction.

Now we are ready to complete the proof. From the exact sequence of $\frac{1}{e} \mathbb{Z}$-graded modules

$$
0 \rightarrow \overline{\mathcal{S}}\left(\frac{1}{e}\right) \stackrel{u^{-1}}{\longrightarrow} \overline{\mathcal{S}} \rightarrow \mathcal{E} \rightarrow 0
$$

we obtain a long exact sequence

$$
\cdots \longrightarrow H_{J}^{1}(\mathcal{E}) \longrightarrow H_{J}^{2}\left(\overline{\mathcal{S}}\left(\frac{1}{e}\right)\right) \stackrel{u^{-1}}{\longrightarrow} H_{J}^{2}(\overline{\mathcal{S}}) \longrightarrow \cdots .
$$

Suppose that $a \in\left[H_{J}^{2}(\overline{\mathcal{S}})\right]_{n}=\left[H_{J}^{2}\left(\overline{\mathcal{S}}\left(\frac{1}{e}\right)\right)\right]_{n-\frac{1}{e}}$ for some rational number $n \leq 0$ and that $a \neq 0$. Let $l$ be the smallest non-negative integer such that $\left(u^{-1}\right)^{l} a=0$. Such integer $l$ exists because $u^{-1}$ belongs to $\sqrt{J}$, and clearly $l>0$ because $a \neq 0$. Therefore $u^{-l+1} a \in$ $\operatorname{ker}\left(\cdot u^{-1}\right) \cap\left[H_{J}^{2}\left(\overline{\mathcal{S}}\left(\frac{1}{e}\right)\right)\right]_{n-\frac{l}{e}}$ and $n-\frac{l}{e}<0$. Now if we show that $\left[\operatorname{ker}\left(\cdot u^{-1}\right)\right]_{m}=0$ for all 
rational numbers $m<0$, then $0=u^{-l+1} a=\left(u^{-1}\right)^{l-1} a$, which contradicts the minimality of $l$. Hence it suffices to prove that $\left[H_{J}^{1}(\mathcal{E})\right]_{m}=0$ for all rational numbers $m<0$.

Since there exists an $\overline{\mathcal{E}}$-regular sequence of length at least 2 in $J \overline{\mathcal{E}}$, we get $H_{J}^{0}(\overline{\mathcal{E}})=H_{J}^{1}(\overline{\mathcal{E}})=$ 0 . The exact sequence $0 \rightarrow \mathcal{E} \rightarrow \overline{\mathcal{E}} \rightarrow \overline{\mathcal{E}} / \mathcal{E} \rightarrow 0$ yields

$$
0=H_{J}^{0}(\overline{\mathcal{E}}) \longrightarrow H_{J}^{0}(\overline{\mathcal{E}} / \mathcal{E}) \stackrel{\cong}{\longrightarrow} H_{J}^{1}(\mathcal{E}) \longrightarrow H_{J}^{1}(\overline{\mathcal{E}})=0 .
$$

Since $\overline{\mathcal{E}}$ is graded by $\frac{1}{e} \mathbb{Z}_{\geq 0}$, hence so is $H_{J}^{0}(\overline{\mathcal{E}} / \mathcal{E}) \subset \overline{\mathcal{E}} / \mathcal{E}$ and we conclude that $\left[H_{J}^{1}(\mathcal{E})\right]_{m} \cong$ $\left[H_{J}^{0}(\overline{\mathcal{E}} / \mathcal{E})\right]_{m}=0$ for all rational numbers $m<0$.

\section{Specialization by Generic Elements of ideals}

We show that the integral closure of any ideal of height at least 2 is compatible with specialization by generic elements. When the ideal is a complete intersection, this theorem is proved in [16, Theorem 1].

Theorem 2.1. Let $R$ be a Noetherian, locally equidimensional, universally catenary ring such that $R_{\text {red }}$ is locally analytically unramified. Let $I=\left(a_{1}, \ldots, a_{n}\right)$ be an $R$-ideal of height at least 2 . Let $R^{\prime}=R\left[z_{1}, \ldots, z_{n}\right]$ be a polynomial ring in the variables $z_{1}, \ldots, z_{n}, I^{\prime}=I R^{\prime}$, and $x=\sum_{i=1}^{n} z_{i} a_{i}$. Then $\overline{I^{\prime} /(x)}=\overline{I^{\prime}} /(x)$.

Proof. We may assume that $R$ is a local ring of dimension $d$ and $I$ is a proper ideal. Let $\mathcal{A}$ and $\mathcal{B}$ be the extended Rees rings of $I^{\prime}$ and $I^{\prime} /(x)$, respectively. Denote $R^{\prime} /(x)$ by $\mathfrak{R}$. Let $\overline{\mathcal{A}}$ and $\overline{\mathcal{B}}$ be the integral closures of $\mathcal{A}$ and $\mathcal{B}$ in $R^{\prime}\left[t, t^{-1}\right]$ and $\mathfrak{R}\left[t, t^{-1}\right]$, respectively. Consider the natural map $\varphi: \overline{\mathcal{A}} / x t \overline{\mathcal{A}} \longrightarrow \overline{\mathcal{B}}$. Let $J$ be the $\mathcal{A}$-ideal $\left(t^{-1}, I^{\prime} t\right)$, which is generated by $t^{-1}$ and by linear forms. The ideal $J$ has height at least 3 . This holds because the $R^{\prime}$-ideal $I^{\prime}$ has height at least 2 and the ring $\mathcal{A}$ is catenary and equidimensional of dimension $d+1$ with a unique maximal homogeneous ideal, which is a maximal ideal.

First we claim that $\varphi_{\mathfrak{p}}$ is an isomorphism for all $\mathfrak{p} \notin V(J)$. Suppose $t^{-1} \notin \mathfrak{p}$. In this case the claim follows because $\varphi_{t^{-1}}$ factors through the natural isomorphisms

$$
(\overline{\mathcal{A}} / x t \overline{\mathcal{A}})_{t^{-1}} \cong \overline{\mathcal{A}}_{t^{-1}} / x t \overline{\mathcal{A}}_{t^{-1}} \cong R^{\prime}\left[t, t^{-1}\right] / x R^{\prime}\left[t, t^{-1}\right] \cong \mathfrak{R}\left[t, t^{-1}\right] \cong \overline{\mathcal{B}}_{t^{-1}}
$$

Suppose $I^{\prime} t \not \subset \mathfrak{p}$. We may assume that $a_{n} t \notin \mathfrak{p}$. One has

$$
\overline{\mathcal{A}}=\overline{R^{\prime}\left[I^{\prime} t, t^{-1}\right]}=\overline{R\left[z_{1}, \ldots, z_{n}\right]\left[I^{\prime} t, t^{-1}\right]} \cong \overline{R\left[I t, t^{-1}\right]}\left[z_{1}, \ldots, z_{n}\right],
$$

where $\overline{R\left[I t, t^{-1}\right]}$ is the integral closure of $R\left[I t, t^{-1}\right]$ in $R\left[t, t^{-1}\right]$. Therefore

$$
\begin{aligned}
(\overline{\mathcal{A}} / x t \overline{\mathcal{A}})_{a_{n} t} & \cong\left(\overline{R\left[I t, t^{-1}\right]}\left[z_{1}, \ldots, z_{n}\right] /(x t)\right)_{a_{n} t} \\
& \cong{\overline{R\left[I t, t^{-1}\right]_{a_{n} t}}}\left[z_{1}, \ldots, z_{n}\right] /\left(\sum_{i=1}^{n-1} \frac{a_{i} z_{i} t}{a_{n} t}+z_{n}\right) \\
& \cong \overline{R\left[I t, t^{-1}\right]_{a_{n} t}}{ }\left[z_{1}, \ldots, z_{n-1}\right] .
\end{aligned}
$$

On the other hand, $(\overline{\mathcal{B}})_{a_{n} t} \subset \mathfrak{R}\left[t, t^{-1}\right]_{a_{n} t}$ and

$$
\mathfrak{R}\left[t, t^{-1}\right]_{a_{n} t} \cong\left(R^{\prime}\left[t, t^{-1}\right] /(x)\right)_{a_{n} t} \cong\left(R^{\prime}\left[t, t^{-1}\right] /(x t)\right)_{a_{n} t} \cong\left(R\left[t, t^{-1}\right]_{a_{n} t}\right)\left[z_{1}, \ldots, z_{n-1}\right] .
$$

Now we have 


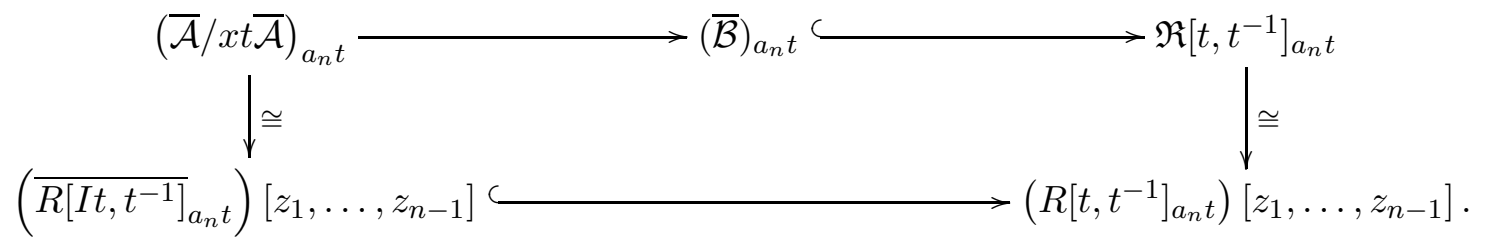

Since $\left({\overline{R\left[I t, t^{-1}\right.}}_{a_{n} t}\right)\left[z_{1}, \ldots, z_{n-1}\right]$ is integrally closed in $\left(R\left[t, t^{-1}\right]_{a_{n} t}\right)\left[z_{1}, \ldots, z_{n-1}\right]$, this proves the claim.

Denote the kernel and cokernel of $\varphi$ by $K$ and $C$, respectively. Our goal is to prove that $C_{1}=0$, which we do by identifying $C$ with a submodule of $H_{J}^{2}(\overline{\mathcal{A}})(-1)$. By the claim above, we have $K=H_{J}^{0}(K)$ and $C=H_{J}^{0}(C)$. Moreover, $H_{J}^{0}(\overline{\mathcal{B}})=0$ because $J$ contains the $\overline{\mathcal{B}}-$ regular element $t^{-1}$. From the exact sequence $0 \rightarrow K \rightarrow \overline{\mathcal{A}} / x t \overline{\mathcal{A}} \stackrel{\varphi}{\longrightarrow} \operatorname{Im}(\varphi) \rightarrow 0$, we obtain $H_{J}^{i}(\overline{\mathcal{A}} / x t \overline{\mathcal{A}}) \cong H_{J}^{i}(\operatorname{Im}(\varphi))$ for all $i \geq 1$. Then, using the exact sequence $0 \rightarrow \operatorname{Im}(\varphi) \rightarrow \overline{\mathcal{B}} \rightarrow$ $C \rightarrow 0$, we get

$$
0=H_{J}^{0}(\overline{\mathcal{B}}) \longrightarrow H_{J}^{0}(C) \longrightarrow H_{J}^{1}(\operatorname{Im}(\varphi)) \cong H_{J}^{1}(\overline{\mathcal{A}} / x t \overline{\mathcal{A}}) .
$$

Therefore $C=H_{J}^{0}(C) \hookrightarrow H_{J}^{1}(\overline{\mathcal{A}} / x t \overline{\mathcal{A}})$.

By Lemma 1.1(c), we have $H_{J}^{1}(\overline{\mathcal{A}})=0$. Hence $0 \rightarrow x t \overline{\mathcal{A}} \rightarrow \overline{\mathcal{A}} \rightarrow \overline{\mathcal{A}} / x t \overline{\mathcal{A}} \rightarrow 0$ gives the exact sequence

Therefore $C \hookrightarrow H_{J}^{2}(x t \overline{\mathcal{A}})$.

$$
0=H_{J}^{1}(\overline{\mathcal{A}}) \longrightarrow H_{J}^{1}(\overline{\mathcal{A}} / x t \overline{\mathcal{A}}) \longrightarrow H_{J}^{2}(x t \overline{\mathcal{A}}) .
$$

Let $L=\operatorname{ann} \overline{\mathcal{A}}_{\mathcal{A}}(x t)$. Notice that $x t$ is a generic element of $I^{\prime} t$. Therefore $L=H_{I^{\prime} t}^{0}(L)$, which implies $H_{I^{\prime} t}^{i}(L)=0$ for all $i \geq 1$. It follows that $H_{I^{\prime} t}^{i}\left(L_{t^{-1}}\right)=0$ for all $i \geq 1$. Since $J=\left(t^{-1}, I^{\prime} t\right)$, we have an exact sequence $([2,8.1 .2])$

$$
H_{I^{\prime} t}^{1}\left(L_{t^{-1}}\right) \longrightarrow H_{J}^{2}(L) \longrightarrow H_{I^{\prime} t}^{2}(L) \longrightarrow H_{I^{\prime} t}^{2}\left(L_{t^{-1}}\right) \longrightarrow H_{J}^{3}(L) \longrightarrow H_{I^{\prime} t}^{3}(L) .
$$

Therefore $H_{J}^{2}(L)=H_{J}^{3}(L)=0$. The exact sequence $0 \rightarrow L \rightarrow \overline{\mathcal{A}}(-1) \stackrel{x t}{\longrightarrow} x t \overline{\mathcal{A}} \rightarrow 0$ now yields an exact sequence

$$
0=H_{J}^{2}(L) \longrightarrow H_{J}^{2}(\overline{\mathcal{A}}(-1)) \longrightarrow H_{J}^{2}(x t \overline{\mathcal{A}}) \longrightarrow H_{J}^{3}(L)=0,
$$

from which we conclude that $C_{n} \hookrightarrow\left[H_{J}^{2}(x t \overline{\mathcal{A}})\right]_{n} \cong\left[H_{J}^{2}(\overline{\mathcal{A}})\right]_{n-1}$. By Theorem 1.2 , we have $\left[H_{J}^{2}(\overline{\mathcal{A}})\right]_{n-1}=0$ for all $n-1 \leq 0$. In particular $C_{1}=0$, that is, $\overline{I^{\prime} /(x)}=\overline{I^{\prime}} /(x)$.

Corollary 2.2. Let $R$ be a Noetherian, locally equidimensional, universally catenary ring such that $R_{\text {red }}$ is locally analytically unramified. Let $I=\left(a_{1}, \ldots, a_{n}\right)$ be an $R$-ideal of height at least 2. Let $R^{\prime}=R\left[z_{1}, \ldots, z_{n}\right], I^{\prime}=I R^{\prime}$, and $x=\sum_{i=1}^{n} z_{i} a_{i}$. Then $\sqrt{(x)} \subset \overline{I^{\prime}}$.

Proof. Let $y \in R^{\prime}$ such that $y^{m} \in(x)$ for some $m$. Then $(y+(x))^{m}=0$ in $R^{\prime} /(x)$. In particular, $y+(x) \in \overline{I^{\prime} /(x)}$. Since $\overline{I^{\prime} /(x)}=\overline{I^{\prime}} /(x)$ by Theorem 2.1, we get $y \in \overline{I^{\prime}}$.

The reader may want to compare the previous corollary to the known result that if $R$ is a Noetherian reduced ring, $I$ an $R$-ideal of grade at least 2, and $x$ a generic element for $I$ as above, then $\sqrt{(x)}=(x)([9$, Theorem (b)]).

The next corollary is a variation and an immediate consequence of Theorem 2.1. We assume $(R, \mathfrak{m})$ is local and we replace the polynomial $\operatorname{ring} R^{\prime}=R\left[z_{1}, \ldots, z_{n}\right]$ by its localization 
$R^{\prime \prime}=R\left(z_{1}, \ldots, z_{n}\right)=R\left[z_{1}, \ldots, z_{n}\right]_{\mathfrak{m} R\left[z_{1}, \ldots, z_{n}\right]}$. In this case, the rings $R$ and $R^{\prime \prime}$ have the same dimension and the generic element $x$ is part of a minimal generating set of the ideal $I R^{\prime \prime}$.

Corollary 2.3. Let $R$ be a Noetherian, equidimensional, universally catenary local ring such that $R_{\text {red }}$ is analytically unramified. Let $I=\left(a_{1}, \ldots, a_{n}\right)$ be an $R$-ideal of height at least 2 . Let $R^{\prime \prime}=R\left(z_{1}, \ldots, z_{n}\right), I^{\prime \prime}=I R^{\prime \prime}$, and $x=\sum_{i=1}^{n} z_{i} a_{i}$. Then $\overline{I^{\prime \prime} /(x)}=\overline{I^{\prime \prime}} /(x)$.

So far we have shown that the integral closure of an ideal is preserved by specialization modulo a generic element. One of the main applications of this result is in proofs using induction on the height of an ideal. For example, under slightly modified assumptions, it yields a direct proof of a well-known theorem on integral closures of ideal powers, due independently to Huneke and Itoh ([11, 4.7], [15, Theorem 1]).

Theorem 2.4. Let $R$ be a Noetherian, locally equidimensional, universally catenary ring such that $R_{\text {red }}$ is locally analytically unramified. Let I be a complete intersection $R$-ideal. Then

$$
\overline{I^{n+1}} \bigcap I^{n}=\bar{I} I^{n} \quad \text { for all } n \geq 0 .
$$

Proof. We may assume that $R$ is local and $g=h \mathrm{t}(I)>0$. Write $G=\bigoplus_{n \geq 0} I^{n} / I^{n+1}$ for the associated graded ring of $I$ and let $\widetilde{G}=\bigoplus_{n \geq 0} \overline{I^{n}} / \overline{I^{n+1}}$. Consider the exact sequence

$$
0 \longrightarrow K \longrightarrow G \otimes_{R} R / \bar{I} \stackrel{\varphi}{\longrightarrow} \widetilde{G}
$$

where $\varphi$ is the natural map and $K$ is its kernel. Notice that the degree $n$ component of $K$ is $K_{n}=\frac{\overline{I^{n+1}} \bigcap I^{n}}{\bar{I} I^{n}}$. We want to show that $K=0$.

We use induction on $g$. First let $g=1$. In this case $I$ is generated by a single regular element $a$. Write $\bar{R}$ for the integral closure of $R$ in its total ring of quotients. Since $\bar{I}=a \bar{R} \cap R$ and $\overline{I^{n+1}}=a^{n+1} \bar{R} \cap R$, we obtain

$$
\overline{I^{n+1}} \cap I^{n}=a^{n+1} \bar{R} \cap a^{n} R=a^{n}(a \bar{R} \cap R)=\bar{I} I^{n} .
$$

It follows that $K=0$ in this case.

Suppose that $g \geq 2$. Let $I=\left(a_{1}, \ldots, a_{g}\right), R^{\prime \prime}=R\left(z_{1}, \ldots, z_{g}\right)$, and $I^{\prime \prime}=I R^{\prime \prime}$. Denote the associated graded ring of $I^{\prime \prime}$ by $G^{\prime \prime}$, set $\widetilde{G^{\prime \prime}}=\bigoplus_{n \geq 0} \overline{\left(I^{\prime \prime}\right)^{n}} / \overline{\left(I^{\prime \prime}\right)^{n+1}}$, and consider the natural exact sequence

$$
0 \longrightarrow K^{\prime \prime} \longrightarrow G^{\prime \prime} \otimes_{R^{\prime \prime}} R^{\prime \prime} / \overline{I^{\prime \prime}} \stackrel{\varphi^{\prime \prime}}{\longrightarrow} \widetilde{G^{\prime \prime}} .
$$

Further we define $x=\sum_{i=1}^{g} z_{i} a_{i} \in I^{\prime \prime}, \mathfrak{R}=R^{\prime \prime} /(x)$, and $\mathfrak{I}=I^{\prime \prime} /(x)$. We wish to apply the induction hypothesis to the ideal $\mathfrak{I}$ of the ring $\mathfrak{R}$. Notice that $\mathfrak{I}$ is a complete intersection ideal of height $g-1$.

We first argue that the condition concerning analytic unramifiedness passes from $R$ to $\mathfrak{R}$. For this part we may assume that $\widehat{R}$ is reduced and it suffices to show that $\widehat{\mathfrak{R}}$ is reduced as well. But indeed, $\widehat{\mathfrak{R}}$ is the completion of the excellent local ring $\widehat{R}\left(z_{1}, \ldots, z_{g}\right) /(x)$, and the latter ring is reduced because $x$ is a generic element for $\widehat{I}$, an ideal of grade at least 2 in the reduced ring $\widehat{R}([9$, Theorem (b)]).

By Corollary 2.3, we have $\overline{\mathfrak{I}}=\overline{I^{\prime \prime}} /(x)$. Let $\mathcal{G}$ be the associated graded ring of $\mathfrak{I}$. We observe that $\mathcal{G} \cong G^{\prime \prime} /\left(x+\left(I^{\prime \prime}\right)^{2}\right)$ because $x$ is part of a regular sequence that generates the ideal $I^{\prime \prime}$. Moreover, the element $x+\left(I^{\prime \prime}\right)^{2}$ is regular on $\widetilde{G^{\prime \prime}}$, hence on $\operatorname{Im}\left(\varphi^{\prime \prime}\right)($ Lemma 1.1(c)). Write 
$\widetilde{\mathcal{G}}=\bigoplus_{n \geq 0} \overline{\mathfrak{I}^{n}} / \overline{\mathfrak{I}^{n+1}}$. We obtain the following commutative diagram with exact rows,

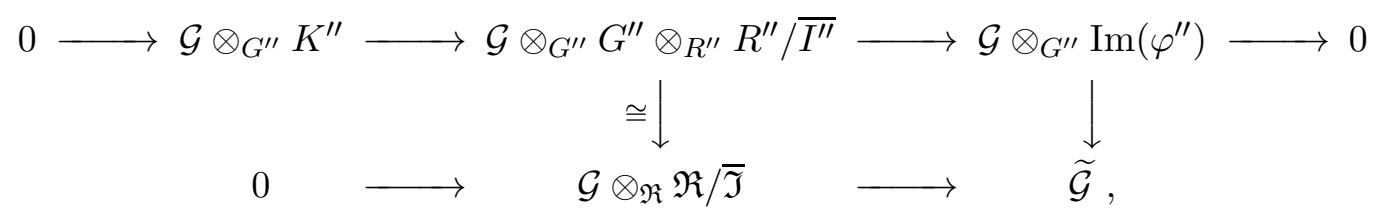

where the vertical isomorphism follows from the equality $\overline{\mathfrak{I}}=\overline{I^{\prime \prime}} /(x)$, the exactness of the first row from the fact that $x+\left(I^{\prime \prime}\right)^{2}$ is regular on $\operatorname{Im}\left(\varphi^{\prime \prime}\right)$, and the exactness of the second row from the induction hypothesis. We conclude that $\mathcal{G} \otimes_{G^{\prime \prime}} K^{\prime \prime}=0$, which gives $K^{\prime \prime}=0$ by the graded Nakayama Lemma. Therefore $K=0$, as asserted.

\section{Modules OVER two Dimensional Regular local RingS}

Notation 3.1. Let $(R, \mathfrak{m})$ be a Noetherian local ring and $E$ a finitely generated torsion-free $R$-module such that $E_{\mathfrak{p}}$ is free for every prime ideal $\mathfrak{p}$ of $R$ with depth $R_{\mathfrak{p}} \leq 1$. In this case $E$ has a rank $e$, which we assume to be positive $([8,2.1])$. Let $U=\sum_{j=1}^{n} R a_{j}$ be a submodule of $E$ so that $E / U$ has grade at least 2, in other words, $U_{\mathfrak{p}}=E_{\mathfrak{p}}$ whenever depth $R_{\mathfrak{p}} \leq 1$. This condition is automatically satisfied if $U$ is a reduction of $E$ because in that case $E / U$ is supported on the non-free locus of $E$. Let $Z=\left\{z_{i j} \mid 1 \leq i \leq e-1,1 \leq j \leq n\right\}$ be a set of indeterminates over $R$. Set

$$
R^{\prime \prime}=R[Z]_{\mathfrak{m} R[Z]}, \quad U^{\prime \prime}=R^{\prime \prime} \otimes_{R} U, \quad E^{\prime \prime}=R^{\prime \prime} \otimes_{R} E, \quad x_{i}=\sum_{j=1}^{n} z_{i j} a_{j}, \quad \text { and } F=\sum_{i=1}^{e-1} R^{\prime \prime} x_{i} .
$$

The module $F$ is a free $R^{\prime \prime}$-module of rank $e-1$ and $E^{\prime \prime} / F$ is torsion-free with rank 1 , hence isomorphic to an $R^{\prime \prime}$-ideal ([23, 3.2]). An $R^{\prime \prime}$-ideal $I$ with $I \cong E^{\prime \prime} / F$ is called a generic Bourbaki ideal of $E$ with respect to $U$ ([23, 3.3]). We refer the reader to [23] for a general discussion of generic Bourbaki ideals, including the fact that a generic Bourbaki ideal is essentially unique ([23, 3.4]). If $E$ has finite projective dimension, then the ideal $I \cong E^{\prime \prime} / F$ can be chosen to have grade at least $2([23,3.2])$. This ideal is uniquely determined by $E$ and the generators $a_{1}, \ldots, a_{n}$ of $U$; we will call it a generic Bourbaki ideal of $E$ with respect to $U$ of grade at least 2. If $U=E$, we simply call it a generic Bourbaki ideal of $E$ of grade at least 2 .

Our objective is to study whether a generic Bourbaki ideal of the integral closure $\bar{E}$ with respect to $E$ is integrally closed. In this section we consider the case of two-dimensional regular local rings. We prove that a generic Bourbaki ideal of grade at least 2 of an integrally closed module is again integrally closed and we express this ideal as a Fitting ideal of the module. Using these facts, we deduce the known results about integrally closed modules over two-dimensional regular local rings from the corresponding classical theory for ideals.

Thus let $(R, \mathfrak{m})$ be a two-dimensional regular local ring. For an $R$-ideal $I$, the order $o(I)$ of $I$ is defined to be the largest integer $r$ such that $I \subset \mathfrak{m}^{r}$. Let $E$ be a finitely generated torsion-free $R$-module with rank $e$ and write $E^{*}=\operatorname{Hom}_{R}(E, R)$. There is a natural embedding $E \subset E^{* *}$, where $E^{* *}$ is a free module of rank $e$ and $E^{* *} / E$ has finite length. One defines the order $o(E)$ of $E$ to be the order of the zeroth Fitting ideal $\operatorname{Fitt}_{0}\left(E^{* *} / E\right)$. We recall that for a presentation $R^{m} \stackrel{\varphi}{\longrightarrow} R^{n} \rightarrow M \rightarrow 0$ of a module $M$, the $i$-th Fitting ideal Fitt $_{i}(M)$ of $M$ is the $R$-ideal $I_{n-i}(\varphi)$ generated by the $(n-i) \times(n-i)$-minors of $\varphi$. This ideal only depends on $M$ and $i$. 
Suppose that the maximal ideal $\mathfrak{m}$ is generated by $a, b$ and let $S=R\left[\frac{\mathfrak{m}}{a}\right]$. Identifying $E^{* *}$ with $R^{e}$, which is contained in $S^{e}$, we let $E S$ denote the $S$-submodule of $S^{e}$ generated by $E$. This module $E S$ is called the transform of $E$ in $S$. It is isomorphic to $E \otimes_{R} S$ modulo $S$-torsion. The $R$-module $E$ is said to be contracted if $E=E S \cap R^{e}$ for some choice of $a$. Finally, we denote the minimal number of generators of $E$ by $\nu(E)$.

We will use these facts about transforms of modules and contracted modules that are proved in [18:

Theorem 3.2. Let $R$ be a 2 -dimensional regular local ring with maximal ideal $\mathfrak{m}=(a, b)$ and let $E$ be a finitely generated torsion-free $R$-module.

(a) If $E$ is integrally closed, then so is the transform $E S$ in $S=R\left[\frac{\mathfrak{m}}{a}\right]$.

(b) If $E$ is integrally closed, then $E$ is contracted.

(c) The module $E$ is contracted if and only if $\nu(E)=o(E)+\operatorname{rank}(E)$.

Proof. Part (a) is proved in [18, 4.6], part (b) in [18, 4.3], and part (c) in [18, 2.5].

Lemma 3.3. Let $R$ be a 2-dimensional regular local ring. Let $E$ be a finitely generated torsionfree $R$-module with rank $e>0$ and let $U$ be a submodule of $E$ so that $E / U$ has finite length. Let $I \cong E^{\prime \prime} / F$ be a generic Bourbaki ideal of $E$ with respect to $U$ of grade at least 2 . Then $I=\operatorname{Fitt}_{1}\left(E^{\prime \prime} / F\right)$ and this ideal is a reduction of $\operatorname{Fitt}_{e}(E) R^{\prime \prime}$.

Proof. We may assume that the residue field of $R$ is infinite. Consider an epimorphism $R^{\prime \prime n} \rightarrow$ $E^{\prime \prime}$ and decompose $R^{\prime \prime n} \cong F \oplus G$, where $G$ is a free $R^{\prime \prime}$-module generated by $n-e+1$ general elements of $R^{\prime \prime n}$. Let $0 \rightarrow R^{\prime \prime n-e} \stackrel{\varphi}{\longrightarrow} F \oplus G \longrightarrow E^{\prime \prime} \rightarrow 0$ be a presentation of $E^{\prime \prime}$, and let $\psi$ be the $n-e+1$ by $n-e$ submatrix of $\varphi$ consisting of the last rows of $\varphi$. Then $\psi$ is a presentation matrix for the module $E^{\prime \prime} / F \cong I$. Since $I$ is an ideal of projective dimension at most 1 and grade at least 2, the Hilbert-Burch Theorem gives that $I=I_{n-e}(\psi)$. Clearly $\operatorname{Fitt}_{1}\left(E^{\prime \prime} / F\right)=I_{n-e}(\psi) \subset I_{n-e}(\varphi)=\operatorname{Fitt}_{e}(E) R^{\prime \prime}$.

It remains to prove that $I_{n-e}(\varphi)$ is integral over $I_{n-e}(\psi)$. Let $-{ }^{*}$ denote dualizing into $R^{\prime \prime}$. We consider the map $F^{*} \oplus G^{*} \stackrel{\varphi^{*}}{\longrightarrow}\left(R^{\prime \prime n-e}\right)^{*}$. Its image $\operatorname{Im}\left(\varphi^{*}\right)$ has rank $n-e$ as a module over $R^{\prime \prime}$, a 2-dimensional local ring with infinite residue field. Therefore $(n-e)+2-1=n-e+1$ general elements of this module generate a reduction $([23,2.3])$. Thus $\varphi^{*}\left(G^{*}\right)$ is a reduction of $\operatorname{Im}\left(\varphi^{*}\right)$. In other words, the colums of the matrix $\varphi^{*}$ are integral over the module generated by the colums of $\psi^{*}$. Now the valuative criterion for integral dependence shows that $I_{n-e}\left(\varphi^{*}\right)$ is integral over $I_{n-e}\left(\psi^{*}\right)$. Thus indeed $I_{n-e}(\varphi)$ is integral over $I_{n-e}(\psi)$.

Lemma 3.4. Let $R$ be a 2-dimensional regular local ring. Let $E$ be a finitely generated torsionfree $R$-module with rank $e>0$ and let $U$ be a submodule of $E$ so that $E / U$ has finite length. Let $I \cong E^{\prime \prime} / F$ be a generic Bourbaki ideal of $E$ with respect to $U$ of grade at least 2 .

(a) The Fitting ideals $\operatorname{Fitt}_{0}\left(E^{* *} / E\right)$ and $\operatorname{Fitt}_{e}(E)$ are equal. Hence $o(E)=o\left(\operatorname{Fitt}_{e}(E)\right)$.

(b) The order of $E$ is equal to the order of $I$.

(c) If $E$ is contracted, then I is contracted.

Proof. (a) Since $E^{* *}$ is free and $E^{* *} / E$ has projective dimension at most 2, the ideals $\operatorname{Fitt}_{0}\left(E^{* *} / E\right)$ and $\operatorname{Fitt}_{e}(E)$ are isomorphic $([4,3.1])$. But both have grade at least 2, which implies that they are equal.

(b) One has $o(E)=o\left(\operatorname{Fitt}_{e}(E)\right)=o\left(\operatorname{Fitt}_{e}(E) R^{\prime \prime}\right)=o(I)$. The first equality holds by part (a) and the second equality is obvious. The third equality follows from Lemma 3.3 , indeed, the 
lemma shows that $\operatorname{Fitt}_{e}(E) R^{\prime \prime}$ is integral over $I$, and any ideal of a regular local ring has the same order as its integral closure because the powers of the maximal ideal are integrally closed. (c) Suppose $E$ is contracted. Then $E^{\prime \prime}$ is contracted so that $\nu\left(E^{\prime \prime}\right)=o\left(E^{\prime \prime}\right)+e$ by Theorem $[3.2(\mathrm{c})$. Hence we obtain

$$
\nu(I) \leq o(I)+1=o\left(E^{\prime \prime}\right)+1=\nu\left(E^{\prime \prime}\right)-e+1 \leq \nu(I)
$$

where the first inequality holds by the Hilbert-Burch Theorem, the first equality follows from part (b), the second equality is explained above, and the last inequality is obvious from the isomorphism $I \cong E^{\prime \prime} / F$. Therefore $I$ is contracted according to Theorem 3.2 (c).

Let $R$ be a 2-dimensional regular local ring with maximal ideal $\mathfrak{m}=(a, b)$ and write $S=$ $R\left[\frac{\mathfrak{m}}{a}\right]$. Let $\mathfrak{N}$ be a maximal ideal of $S$ containing $\mathfrak{m} S$. Then $T=S_{\mathfrak{N}}$ is a 2-dimensional regular local ring and is called a first quadratic transformation of $R$. For an $R$-ideal $I$ of grade at least 2, we can write $I S=a^{r} I^{\sharp}$ for some $S$-ideal $I^{\sharp}$, where $r=o(I)$. We call the localization $\left(I^{\sharp}\right)_{\mathfrak{N}}$ a first quadratic transform of $I$ and denote it by $\widetilde{I}$. This ideal has grade at least $2([24,14.3 .2])$. Let $E$ be a finitely generated torsion-free $R$-module. We call the module $E T=(E S)_{\mathfrak{N}}$ the transform of $E$ in $T$. The next lemma explains how a generic Bourbaki ideal of grade at least 2 behaves under a first quadratic transformation.

Lemma 3.5. Let $(R, \mathfrak{m})$ be a 2-dimensional regular local ring and let $T=R\left[\frac{\mathfrak{m}}{a}\right]_{\mathfrak{N}}$ be a first quadratic transformation of $R$. Let $E$ be a finitely generated torsion-free $R$-module and $U$ a reduction of $E$. Let $I \subset R^{\prime \prime}$ be a generic Bourbaki ideal of $E$ with respect to $U$ of grade at least 2 and write $T^{\prime \prime}=R^{\prime \prime}\left[\frac{\mathfrak{m}}{a}\right]_{\mathfrak{N} R^{\prime \prime}\left[\frac{\mathfrak{m}}{a}\right]}$. Then the first quadratic transform of $I$ in $T^{\prime \prime}$ is a generic Bourbaki ideal of ET with respect to UT of grade at least 2.

Proof. We use the notation of 3.1 in particular, $R^{\prime \prime}=R[Z]_{\mathfrak{m} R[Z]}$ and $I \cong E^{\prime \prime} / F$. Notice that $T^{\prime \prime}=T[Z]_{\mathfrak{N} T[Z]}$, the module $U T$ is a reduction of $E T$, and $F T^{\prime \prime}$ is a generic $(e-1)$-generated submodule of $U T^{\prime \prime}$. Therefore $E^{\prime \prime} T^{\prime \prime} / F T^{\prime \prime}$ is a torsion-free $T^{\prime \prime}$-module of rank 1 . There are surjective homomorphisms of $T^{\prime \prime}$-modules of rank 1 ,

$$
E^{\prime \prime} T^{\prime \prime} / F T^{\prime \prime} \leftarrow\left(E^{\prime \prime} / F\right) \otimes_{R^{\prime \prime}} T^{\prime \prime} \rightarrow I T^{\prime \prime} .
$$

Since the outer two modules are torsion-free, one obtains an isomorphism $E^{\prime \prime} T^{\prime \prime} / F T^{\prime \prime} \cong I T^{\prime \prime}$. As $I T^{\prime \prime} \cong \widetilde{I}$ and the latter ideal has grade at least 2 , it follows that $\widetilde{I}$ is a generic Bourbaki ideal of $E T$ with respect to $U T$ of grade at least 2 .

Theorem 3.6. Let $R$ be a 2-dimensional regular local ring. Let $E$ be a finitely generated torsion-free $R$-module with rank $e>0$ and let $U$ be a reduction of $E$. Then $E$ is integrally closed if and only if any generic Bourbaki ideal of $E$ with respect to $U$ is integrally closed.

Proof. It will follow from Proposition 4.6 that $E$ is integrally closed if some generic Bourbaki ideal of $E$ with respect to $U$ is integrally closed. To prove the converse suppose that $E$ is integrally closed. We may assume that the residue field of $R$ is infinite. We use the notation of 3.1 and we write $\mathfrak{n}=\mathfrak{m} R^{\prime \prime}=(a, b)$ for the maximal ideal of $R^{\prime \prime}$. Furthermore, let $I \cong E^{\prime \prime} / F$ be a generic Bourbaki ideal of $E$ with respect to $U$ of grade at least 2 .

In order to prove that any generic Bourbaki ideal of $E$ with respect to $U$ is integrally closed, it suffices to show that $I$ is integrally closed ([23, 3.4(a)]). We use induction on the multiplicity $e(I)$ of $I$. If $e(I)=0$, then $I=R^{\prime \prime}$ and we are done. Suppose $e(I) \geq 1$. Since $E$ is integrally closed, $E$ is contracted according to Theorem $3.2(\mathrm{~b})$. Therefore $I$ is contracted by Lemma 3.4(c). Hence we have $I=I S^{\prime \prime} \cap R^{\prime \prime}$ for some $S^{\prime \prime}=R^{\prime \prime}\left[\frac{\mathfrak{n}}{a}\right]$. Since $R / \mathfrak{m}$ is infinite, we 
may choose $a$ to be a general element of $\mathfrak{m}([24,14.2 .2])$, in particular, $a \in \mathfrak{m}$ and $S^{\prime \prime}=R^{\prime \prime}\left[\frac{\mathfrak{m}}{a}\right]$. As $I$ is contracted from $I S^{\prime \prime}$, it suffices to prove that $I S^{\prime \prime}$ is integrally closed. Write $I^{\sharp}=\frac{1}{a^{r}} I S^{\prime \prime}$, where $r=o(I)$. We need to show that $\left(I^{\sharp}\right)_{\mathfrak{N}^{\prime \prime}}$ is integrally closed for every maximal ideal $\mathfrak{N}^{\prime \prime}$ containing $I^{\sharp}$. Notice that any such $\mathfrak{N}^{\prime \prime}$ contains $\mathfrak{m}$. For simplicity, write $T^{\prime \prime}=S_{\mathfrak{N}^{\prime \prime}}^{\prime \prime}, \widetilde{I}=\left(I^{\sharp}\right)_{\mathfrak{N}^{\prime \prime}}$, $S=R\left[\frac{\mathfrak{m}}{a}\right], \mathfrak{N}=\mathfrak{N}^{\prime \prime} \cap S$, and $T=S_{\mathfrak{N}}$.

First assume that $\operatorname{dim} T \geq 2=\operatorname{dim} T^{\prime \prime}$. Since $S^{\prime \prime}$ is a ring of fractions of the polynomial ring $S[Z]$, it follows that $\mathfrak{N}^{\prime \prime}$ is extended from $\mathfrak{N}$ in this case, hence $T^{\prime \prime}=S_{\mathfrak{N} S^{\prime \prime}}^{\prime \prime}$. Thus Lemma 3.5 shows that $\widetilde{I}$ is a generic Bourbaki ideal of ET with respect to $U T$ of grade at least 2. The transform $E T$ of the integrally closed module $E$ is again integrally closed according to Theorem 3.2(a). Moreover, [12, 3.6] gives $e(\widetilde{I})<e(I)$. Now our induction hypothesis implies that $\widetilde{I}$ is integrally closed, as asserted.

Next suppose that $\operatorname{dim} T \leq 1$. We will show that the $T^{\prime \prime}$-module $E T^{\prime \prime} / F T^{\prime \prime}$ is cyclic in this case. Since this module maps onto $\widetilde{I}$, it will follow that $\widetilde{I}$ is principal, hence integrally closed. Now, since $\mathfrak{m} \subset \mathfrak{N}$, we see that $\operatorname{dim} T=1$. Thus $T$ is a discrete valuation ring. In particular, the local map $T \rightarrow T^{\prime \prime}=T[Z]_{\mathfrak{M}}$ is flat with a one-dimensional closed fiber $K[Z]_{\mathfrak{M} K[Z]}$, where $\mathfrak{M}$ is a prime ideal of $T[Z]$ and $K$ stands for the residue field of $T$. Moreover, the $T$-module $E T$ is free of rank $e$ and therefore $U T=E T$. After changing the generating sequence $a_{1}, \ldots, a_{n}$ of the module $U T=E T$ and applying a linear change of variables of the polynomial ring $T[Z]$, we may assume that $a_{1}, \ldots, a_{e}$ form a basis of $E T$ and $a_{e+1}=\ldots=a_{n}=0$. Thus $\operatorname{Fitt}_{1}\left(E T^{\prime \prime} / F T^{\prime \prime}\right)=I_{e-1}(\varphi) T^{\prime \prime}$, where $\varphi$ is the $e-1$ by $e$ matrix consisting of the first $e$ columns of the matrix of variables $\left(z_{i j}\right)$. This ideal has height at least two when extended to $K[Z]_{\mathfrak{M} K[Z]}$, a ring of dimension one. We conclude that the extended ideal is the unit ideal and hence so is $\operatorname{Fitt}_{1}\left(E T^{\prime \prime} / F T^{\prime \prime}\right)$. Thus the $T^{\prime \prime}$-module $E T^{\prime \prime} / F T^{\prime \prime}$ is cyclic, as asserted.

Corollary 3.7. Let $R$ be a 2-dimensional regular local ring. Let $E$ be a finitely generated torsion-free $R$-module with rank $e>0$ and let $U$ be a reduction of $E$. Let $I \subset R^{\prime \prime}$ be a generic Bourbaki ideal of $E$ with respect to $U$ of grade at least 2. If $E$ is integrally closed, then $I=\operatorname{Fitt}_{e}(E) R^{\prime \prime}$ and $\operatorname{Fitt}_{e}(E)$ is integrally closed.

Proof. The assertion follows from Lemma 3.3 and Theorem 3.6.

That the ideal $\operatorname{Fitt}_{e}(E)$ in the corollary above is integrally closed can also be seen from [18, 5.4] by way of Lemma 3.4(a). The next result about integrally closed modules over 2dimensional regular local rings has been proved in [18, 5.2] and [17, 4.1]. Here we deduce it from the corresponding statements for ideals, using Theorem 3.6.

Corollary 3.8. Let $R$ be a 2-dimensional regular local ring. Let $E$ be a finitely generated torsion-free $R$-module. Suppose $E$ is integrally closed. Then

(a) The module $E$ is normal.

(b) $E^{2}=U E$ for every reduction $U$ of $E$.

(c) The Rees algebra $\mathcal{R}(E)$ is Cohen-Macaulay.

(d) Let $G$ be a finitely generated torsion-free integrally closed $R$-module. Then EG= $\left(E \otimes_{R} G\right) / \tau\left(E \otimes_{R} G\right)$ is integrally closed, where $\tau(-)$ denotes $R$-torsion.

Proof. Let $U$ be any reduction of $E$. By Theorem 3.6, a generic Bourbaki ideal of $E$ with respect to $U$ of grade at least 2 is integrally closed. Now part (a) follows from [24, 14.4.4] and [23, 3.5(a)(ii)], part (b) from [19, 5.5] and [23, 3.5(b),(c)], and part (c) from [14, 3.2] and [23, $3.5(\mathrm{a})(\mathrm{i})]$. Finally for part (d), notice that $(E \oplus G)^{2}$ is integrally closed by part (a). Since 
$E G$ is a direct summand of $(E \oplus G)^{2}=E^{2} \oplus E G \oplus G^{2}$, the module $E G$ is integrally closed as well.

\section{Specialization By Generic Elements of modules}

In this section we are going to show, under fairly general assumptions, that integral closures of modules are compatible with factoring out generic elements. Some of the proofs here will rely on the results for 2-dimensional regular local rings that were obtained in the previous section.

Lemma 4.1. Let $R$ be a Noetherian ring and $E$ a finitely generated torsion-free $R$-module having a rank e. Suppose that $E_{\mathfrak{p}}$ is free whenever depth $R_{\mathfrak{p}} \leq 1$. Then there exists an embedding $E \subset R^{e}$ such that $\left(R^{e} / E\right)_{\mathfrak{p}}$ is cyclic whenever depth $R_{\mathfrak{p}} \leq 1$.

Proof. We write $-^{*}=\operatorname{Hom}_{R}(-, R)$. There is an epimorphism $\left(R^{m}\right)^{*} \rightarrow E^{*}$, which induces an embedding $E^{* *} \hookrightarrow R^{m}$. Since the natural homomorphism $E \rightarrow E^{* *}$ is injective, we obtain an exact sequence $0 \rightarrow E \rightarrow R^{m} \rightarrow C \rightarrow 0$. For every prime ideal $\mathfrak{p}$ with depth $R_{\mathfrak{p}} \leq 1$, the $R_{\mathfrak{p}}$-modules $E_{\mathfrak{p}}$ and $E_{\mathfrak{p}}^{*}$ are free of rank $e$, hence $C_{\mathfrak{p}}$ is free of rank $m-e$. By basic element theory there exists a basis $\alpha_{1}, \ldots, \alpha_{m}$ of $R^{m}$ such that for the $R$-modules $G \subset F$ generated by the images in $C$ of $\alpha_{1}, \ldots, \alpha_{m-e-1}$ and of $\alpha_{1}, \ldots, \alpha_{m-e}$, the $R$-module $C / F$ has rank 0 and $(C / G)_{\mathfrak{p}} \cong R_{\mathfrak{p}}$ whenever depth $R_{\mathfrak{p}} \leq 1$ ([20,1.1]). In particular, $F$ is a free $R$-module of rank $m-e$. Thus we obtain the following commutative diagram with exact rows and columns,

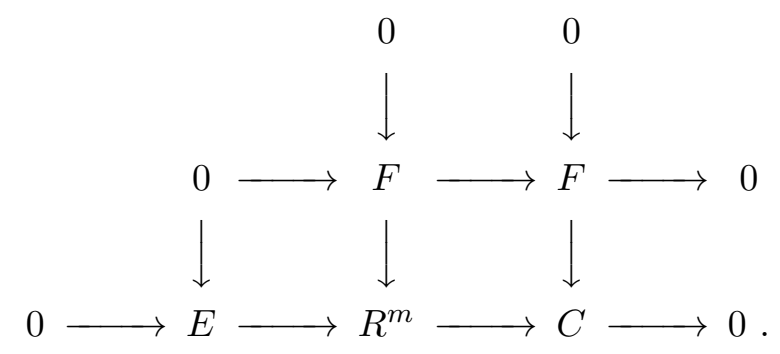

Since $F$ is a free direct summand of $R^{m}$ of rank $m-e$, the Snake Lemma yields an exact sequence $0 \rightarrow E \rightarrow R^{e} \rightarrow C / F \rightarrow 0$. For any prime ideal $\mathfrak{p}$ with depth $R_{\mathfrak{p}} \leq 1$, we have

$$
\nu\left(\left(R^{e} / E\right)_{\mathfrak{p}}\right)=\nu\left((C / F)_{\mathfrak{p}}\right) \leq \nu\left((C / G)_{\mathfrak{p}}\right)=1 .
$$

Let $R$ be a Noetherian ring and $E$ a finitely generated $R$-module having a rank. Recall that $E$ is said to be of linear type if the natural map from the symmetric algebra $\mathcal{S}(E)$ onto the Rees algebra $\mathcal{R}(E)$ is an isomorphism, or equivalently, if $\mathcal{S}(E)$ is $R$-torsion-free.

Lemma 4.2. Let $R$ be a Noetherian ring and let $E=\sum_{i=1}^{n} R a_{i}$ be an $R$-module having a rank $e \geq 2$. Let $E \subset R^{e}$ be an embedding such that $\nu\left(\left(R^{e} / E\right)_{\mathfrak{p}}\right) \leq e-1$ whenever depth $R_{\mathfrak{p}} \leq 1$. Let $R^{\prime}=R\left[z_{1}, \ldots, z_{n}\right], E^{\prime}=R^{\prime} \otimes_{R} E$, and $x=\sum_{i=1}^{n} z_{i} a_{i}$. Then $R^{\prime e} / R^{\prime} x$ is of linear type.

Proof. The embedding $E \subset R^{e}$ and the chosen generators $a_{1}, \ldots, a_{n}$ of $E$ induce an exact sequence $R^{n} \stackrel{\psi}{\longrightarrow} R^{e} \rightarrow R^{e} / E \rightarrow 0$. Notice that grade $I_{1}(\psi) \geq 2$ because $\nu\left(\left(R^{e} / E\right)_{\mathfrak{p}}\right) \leq e-1$ for every $\mathfrak{p} \in \operatorname{Spec}(R)$ with depth $R_{\mathfrak{p}} \leq 1$. Consider the column vector $\Phi=\psi\left[\begin{array}{c}z_{1} \\ \vdots \\ z_{n}\end{array}\right]$. Since 
grade $I_{1}(\Phi) \geq 1$ we have an exact sequence $0 \rightarrow R^{\prime} \stackrel{\Phi}{\longrightarrow} R^{\prime e} \longrightarrow R^{\prime e} / R^{\prime} x \rightarrow 0$. As $R^{\prime e} / R^{\prime} x$ has projective dimension at most 1 , in order to prove that this module is of linear type, it suffices to show that grade $I_{1}(\Phi) \geq 2$ ([1, Proposition 4]).

Suppose there exists a prime ideal $\mathfrak{P} \in \operatorname{Spec}\left(R^{\prime}\right)$ containing $I_{1}(\Phi)$ such that depth $R_{\mathfrak{P}}^{\prime} \leq 1$. Let $\mathfrak{p}=\mathfrak{P} \cap R$. We have depth $R_{\mathfrak{p}} \leq 1$. Then since grade $I_{1}(\psi) \geq 2$, we get $I_{1}(\psi)_{\mathfrak{p}}=R_{\mathfrak{p}}$. After elementary row and column operations on the matrix $\psi_{\mathfrak{p}}$ and a linear change of variables of the polynomial ring $R_{\mathfrak{p}}\left[z_{1}, \ldots, z_{n}\right]$, we may assume that

$$
\psi_{\mathfrak{p}}=\left[\begin{array}{cccc}
1 & 0 & \cdots & 0 \\
0 & b_{22} & \cdots & b_{2 n} \\
0 & \vdots & \cdots & \vdots \\
0 & b_{e 2} & \cdots & b_{e n}
\end{array}\right] \quad \text { and } \quad \Phi_{\mathfrak{P}}=\left[\begin{array}{c}
z_{1} \\
b_{22} z_{2}+\cdots+b_{2 n} z_{n} \\
\vdots \\
b_{e 2} z_{2}+\cdots+b_{e n} z_{n}
\end{array}\right]
$$

Since $E=\operatorname{Im}(\psi)$ has rank $e \geq 2$, we have grade $I_{2}(\psi) \geq 1$. Therefore the ideal

$$
\left(b_{22} z_{2}+\cdots+b_{2 n} z_{n}, \ldots, b_{e 2} z_{2}+\cdots+b_{e n} z_{n}\right)
$$

in $R_{\mathfrak{p}}\left[z_{2}, \ldots, z_{n}\right]$ has grade at least 1 . It follows that the $R_{\mathfrak{P}}^{\prime}$-ideal $I_{1}\left(\Phi_{\mathfrak{P}}\right)$ has grade at least 2 , which is impossible since this ideal is contained in $\mathfrak{P} R_{\mathfrak{P}}^{\prime}$ and depth $R_{\mathfrak{P}}^{\prime} \leq 1$.

Lemma 4.2 and the next Theorem 4.3 require an embedding $E \subset R^{e}$ such that $\nu\left(\left(R^{e} / E\right)_{\mathfrak{p}}\right) \leq$ $e-1$ for any $\mathfrak{p} \in \operatorname{Spec}(R)$ with depth $R_{\mathfrak{p}} \leq 1$. Such an embedding exists by Lemma 4.1 if $e \geq 2$ and the assumptions of the lemma are satisfied. Now we are ready to prove the module analogue of Theorem 2.1.

Theorem 4.3. Let $R$ be a Noetherian, locally equidimensional, universally catenary ring such that $R_{\text {red }}$ is locally analytically unramified. Let $E=\sum_{i=1}^{n} R a_{i}$ be an $R$-module having a rank $e \geq 2$. Let $E \subset R^{e}$ be an embedding such that $\nu\left(\left(R^{e} / E\right)_{\mathfrak{p}}\right) \leq e-1$ whenever depth $R_{\mathfrak{p}} \leq 1$. Let $R^{\prime}=R\left[z_{1}, \ldots, z_{n}\right], E^{\prime}=R^{\prime} \otimes_{R} E$, and $x=\sum_{i=1}^{n} z_{i} a_{i}$. Let $\overline{E^{\prime}}$ and $\overline{E^{\prime} / R^{\prime} x} R^{\prime e} / R^{\prime} x$ denote the integral closure of $E^{\prime}$ in $R^{\prime e}$ and that of $E^{\prime} / R^{\prime} x$ in $R^{\prime e} / R^{\prime} x$. Then

$$
\overline{E^{\prime} / R^{\prime} x} R^{\prime e} / R^{\prime} x=\overline{E^{\prime}} / R^{\prime} x \text {. }
$$

Proof. We may assume that $(R, \mathfrak{m})$ is a local ring of dimension $d$. The embedding $E \subset R^{e}$ identifies $E$ with a set of linear forms in the polynomial ring $S=\mathcal{S}\left(R^{e}\right)=R\left[t_{1}, \ldots, t_{e}\right]$. Let $I$ be the $S$-ideal generated by $E$. The symmetric algebra $\mathcal{S}\left(R^{e} / E\right)$ of $R^{e} / E$ is $S / I$, and from [13, 2.6] we obtain

$$
\operatorname{ht}(I)=d+e-\operatorname{dim}\left(\mathcal{S}\left(R^{e} / E\right)\right)=\min _{\mathfrak{p} \in \operatorname{Spec}(R)}\left\{e+\operatorname{ht}(\mathfrak{p})-\nu\left(\left(R^{e} / E\right)_{\mathfrak{p}}\right)\right\} .
$$

For any $\mathfrak{p} \in \operatorname{Spec}(R)$, we have

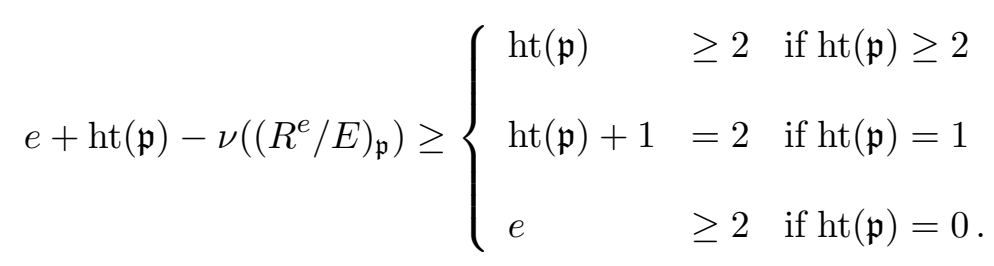

Therefore the $S$-ideal $I$ generated by $E$ has height at least 2. Let $S^{\prime}=S\left[z_{1}, \ldots, z_{n}\right], I^{\prime}=I S^{\prime}$, and $x=\sum_{i=1}^{n} z_{i} a_{i}$. We have $\overline{I^{\prime}} /(x)=\overline{I^{\prime} /(x)} \subset S^{\prime} /(x)$ by Theorem 2.1. The theorem applies because $S_{\text {red }}$ is locally analytically unramified; in fact, since $I$ is a homogeneous ideal, it suffices 
that the localization $\left(S_{\mathrm{red}}\right)_{\left(\mathfrak{m}, t_{1}, \ldots, t_{e}\right)}$ is analytically unramified; but the completion of this ring is $\widehat{R_{\text {red }}}\left[\left[t_{1}, \ldots, t_{e}\right]\right]$, which is reduced.

The embedding $E^{\prime} \subset R^{\prime e}$ defines the Rees algebra $\mathcal{R}\left(E^{\prime}\right)$ as a subalgebra of the polynomial ring $S^{\prime}=\mathcal{S}\left(R^{\prime e}\right)=R^{\prime}\left[t_{1}, \ldots, t_{e}\right]$. Notice that $I^{\prime}$ is the $S^{\prime}$ ideal generated by $E^{\prime}$. By [10, 1.2$]$, we have $\overline{E^{\prime}}=\left[\overline{I^{\prime}}\right]_{1}$. Therefore we obtain

$$
\left[\overline{I^{\prime} /(x)}\right]_{1}=\left[\overline{I^{\prime}} /(x)\right]_{1}=\overline{E^{\prime}} / R^{\prime} x \subset{\overline{E^{\prime} / R^{\prime} x}}^{R^{\prime e} / R^{\prime} x} \subset R^{\prime e} / R^{\prime} x .
$$

It remains to show that $\overline{E^{\prime} / R^{\prime} x} R^{\prime e} / R^{\prime} x \subset\left[\overline{I^{\prime} /(x)}\right]_{1}$. Since the module $R^{\prime e} / R^{\prime} x$ is of linear type (Lemma 4.2), we have

$$
\mathcal{R}\left(R^{\prime e} / R^{\prime} x\right) \cong \mathcal{S}\left(R^{\prime e} / R^{\prime} x\right) \stackrel{\Phi}{\longrightarrow} S^{\prime} /(x),
$$

where $\Phi$ is the natural map induced by the identity on $R^{\prime e} / R^{\prime} x$. Let $u \in \overline{E^{\prime} / R^{\prime} x} R^{\prime e} / R^{\prime} x$ $R^{\prime e} / R^{\prime} x$. Then there is an equation in $\mathcal{R}\left(R^{\prime e} / R^{\prime} x\right)$ of the form

$$
u^{m}+a_{1} u^{m-1}+\cdots+a_{m}=0, \quad a_{i} \in\left(E^{\prime} / R^{\prime} x\right)^{i} .
$$

Applying $\Phi$ to this equation, it converts into an equation of integrality of $u \in S^{\prime} /(x)$ over the ideal $I^{\prime} /(x)$. Therefore we obtain $u \in\left[\overline{I^{\prime} /(x)}\right]_{1}$.

In the remainder of this section, we are going to strengthen Theorem 4.3 under more stringent hypotheses, in that we replace $\overline{E^{\prime} / R^{\prime} x} R^{\prime e} / R^{\prime} x$ by $\overline{E^{\prime} / R^{\prime} x}$, the integral closure of $E^{\prime} / R^{\prime} x$ in a free module of the same rank. Recall that this integral closure does not depend on the choice of embedding into a free module (of arbitrary rank even) if the ring is normal.

Theorem 4.4. Let $R$ be a Noetherian, universally catenary, normal ring that is locally analytically unramified. Let $E=\sum_{i=1}^{n} R a_{i}$ be a torsion-free $R$-module having a rank $e \geq 2$. Let $R^{\prime}=R\left[z_{1}, \ldots, z_{n}\right], E^{\prime}=R^{\prime} \otimes_{R} E$, and $x=\sum_{i=1}^{n} z_{i} a_{i}$. Suppose that for every $\mathfrak{p} \in \operatorname{Spec}(R)$ with depth $R_{\mathfrak{p}} \leq 2$ either $E_{\mathfrak{p}}$ has a nontrivial free direct summand or $R_{\mathfrak{p}}$ is regular. Then the $R^{\prime}$-module $E^{\prime} / R^{\prime} x$ is torsion-free and $\overline{E^{\prime} / R^{\prime} x}=\overline{E^{\prime}} / R^{\prime} x$.

Proof. By Lemma 4.1, there exists an embedding $E \hookrightarrow R^{e}$ such that $\nu\left(\left(R^{e} / E\right)_{\mathfrak{p}}\right) \leq e-1$ whenever depth $R_{\mathfrak{p}} \leq 1$. By Lemma 4.2, $R^{\prime e} / R^{\prime} x$ is a torsion-free module of rank $e-1$. Therefore $E^{\prime} / R^{\prime} x$ is torsion-free and there exists an embedding $R^{\prime e} / R^{\prime} x \hookrightarrow R^{\prime e-1}$. Consider the following diagram,

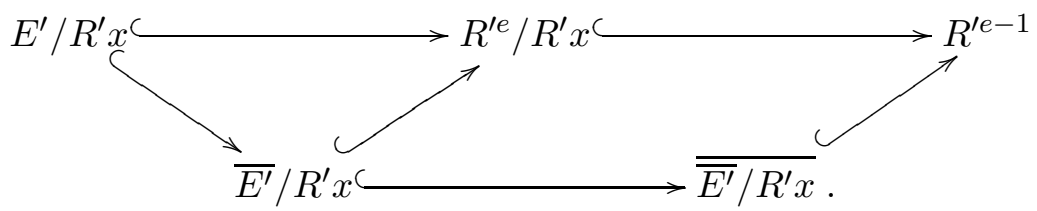

Let $\mathfrak{E}$ denote $\overline{E^{\prime}} / R^{\prime} x$. By Theorem 4.3 , we have

$$
\mathfrak{E}=\overline{\mathfrak{E}} \cap\left(R^{\prime e} / R^{\prime} x\right) .
$$

Therefore we obtain

$$
\overline{\mathfrak{E}} / \mathfrak{E} \subset R^{\prime e-1} /\left(R^{\prime e} / R^{\prime} x\right) .
$$


Since the projective dimension of $R^{\prime e-1} /\left(R^{\prime e} / R^{\prime} x\right)$ is at most 2 , we have depth $R_{\mathfrak{P}}^{\prime} \leq 2$ for every associated prime $\mathfrak{P} \in \operatorname{Ass}_{R^{\prime}}(\overline{\mathfrak{E}} / \mathfrak{E})$. Hence it suffices to show that $\mathfrak{E}_{\mathfrak{P}}$ is integrally closed for every prime ideal $\mathfrak{P}$ with depth $R_{\mathfrak{P}}^{\prime} \leq 2$. Let $\mathfrak{p}=\mathfrak{P} \cap R$.

We first notice that we may replace the generating sequence $a_{1}, \ldots, a_{n}$ of $E_{\mathfrak{p}}$ by any other generating sequence of the same length. Indeed, any two such sequences are related by an invertible matrix with entries in $R_{\mathfrak{p}}$ and the inverse of such a matrix defines a linear change of variables of the polynomial ring $R_{\mathfrak{p}}\left[z_{1}, \ldots, z_{n}\right]$.

Now suppose that depth $R_{\mathfrak{p}} \leq 1$. Then $E_{\mathfrak{p}}$ is free and we may choose $a_{1}, \ldots, a_{e}$ to be a basis and $a_{e+1}=\ldots=a_{n}=0$. The freeness also gives $E_{\mathfrak{p}}=\bar{E}_{\mathfrak{p}}$. If $z_{1}, \ldots, z_{e}$ are not all in $\mathfrak{P}$, then

$$
\mathfrak{E}_{\mathfrak{P}}=\left(E^{\prime} / R^{\prime} x\right)_{\mathfrak{P}}=\left(R_{\mathfrak{P}}^{\prime} a_{1} \oplus \cdots \oplus R_{\mathfrak{P}}^{\prime} a_{e}\right) / R_{\mathfrak{P}}^{\prime}\left(z_{1} a_{1}+\cdots+z_{e} a_{e}\right) \cong\left(R_{\mathfrak{P}}^{\prime}\right)^{e-1} .
$$

Thus $\mathfrak{E}_{\mathfrak{P}}$ is free in this case, hence integrally closed. Otherwise, since $e \geq 2$ and depth $R_{\mathfrak{P}}^{\prime} \leq 2$, we conclude that $e=2$ and $\left(z_{1}, z_{2}\right) R_{\mathfrak{P}}^{\prime}=\mathfrak{P} R_{\mathfrak{P}}^{\prime}$. Therefore

$$
\mathfrak{E}_{\mathfrak{P}}=\left(R_{\mathfrak{P}}^{\prime} a_{1} \oplus R_{\mathfrak{P}}^{\prime} a_{2}\right) / R_{\mathfrak{P}}^{\prime}\left(z_{1} a_{1}+z_{2} a_{2}\right) \cong\left(z_{1}, z_{2}\right) R_{\mathfrak{P}}^{\prime},
$$

which is the maximal ideal. Hence again $\mathfrak{E}_{\mathfrak{P}}$ is integrally closed.

Next consider the case depth $R_{\mathfrak{p}}=2$. We have depth $R_{\mathfrak{P}}^{\prime} \leq 2=\operatorname{depth} R_{\mathfrak{p}}$. Since $R^{\prime}$ is a polynomial ring over $R$, we see that $\mathfrak{P}=\mathfrak{p} R^{\prime}$, hence $R_{\mathfrak{P}}^{\prime}=R_{\mathfrak{p}}\left(z_{1}, \ldots, z_{n}\right)$. Now suppose $E_{\mathfrak{p}}$ has a nontrivial free summand. In this case we may assume that $a_{1}=(1,0)$ in $R_{\mathfrak{p}} \oplus L=E_{\mathfrak{p}}$ and that $a_{2}, \ldots, a_{n}$ generate $L$. Since $z_{1}$ is a unit in $R_{\mathfrak{P}}^{\prime}$, we obtain

$$
\mathfrak{E}_{\mathfrak{P}}=\left(R_{\mathfrak{P}}^{\prime} \oplus R_{\mathfrak{P}}^{\prime} \otimes_{R_{\mathfrak{p}}} \bar{L}\right) / R_{\mathfrak{P}}^{\prime}\left(z_{1}, z_{2} a_{2}+\cdots+z_{n} a_{n}\right) \cong R_{\mathfrak{P}}^{\prime} \otimes_{R_{\mathfrak{p}}} \bar{L} .
$$

Therefore $\mathfrak{E}_{\mathfrak{P}}$ is integrally closed. Next suppose $R_{\mathfrak{p}}$ is regular, necessarily of dimension 2 . In this case $R_{\mathfrak{P}}^{\prime}=R_{\mathfrak{p}}\left(z_{1}, \ldots, z_{n}\right)$ is a 2-dimensional regular local ring as well. Since $R_{\mathfrak{P}}^{\prime}=$ $R_{\mathfrak{p}}\left(z_{1}, \ldots, z_{n}\right)$, it follows that a generic Bourbaki ideal of $\mathfrak{E}_{\mathfrak{P}}$ with respect to $\left(E^{\prime} / R^{\prime} x\right)_{\mathfrak{P}}$ is also a generic Bourbaki ideal of $\bar{E}_{\mathfrak{p}}$ with respect to $E_{\mathfrak{p}}$. Applying Theorem 3.6 twice, now shows that $\mathfrak{E}_{\mathfrak{P}}$ is integrally closed, as asserted.

Corollary 4.5. Let $R$ be a Noetherian, universally catenary, normal local ring that is analytically unramified. Let $E$ be a finitely generated torsion-free $R$-module with rank e $>0$. Suppose that for every $\mathfrak{p} \in \operatorname{Spec}(R)$ with depth $R_{\mathfrak{p}} \leq 2$ either $E_{\mathfrak{p}}$ has a free direct summand of rank $e-1$ or $R_{\mathfrak{p}}$ is regular. Then a generic Bourbaki ideal of $\bar{E}$ with respect to $E$ is integrally closed.

Proof. We may assume that $e \geq 2$. Let $E=\sum_{i=1}^{n} R a_{i}, R^{\prime \prime}=R\left(z_{1}, \ldots, z_{n}\right), E^{\prime \prime}=R^{\prime \prime} \otimes_{R} E$, and $x=\sum_{i=1}^{n} z_{i} a_{i}$. By Theorem 4.4, $E^{\prime \prime} / R^{\prime \prime} x$ is torsion-free. Let $\mathfrak{P} \in \operatorname{Spec}\left(R^{\prime \prime}\right)$ such that depth $R_{\mathfrak{P}}^{\prime \prime} \leq 2$. Once we show that either $\left(E^{\prime \prime} / R^{\prime \prime} x\right)_{\mathfrak{P}}$ has a free summand of rank $e-2$ or $R_{\mathfrak{P}}^{\prime \prime}$ is regular, then the assertion of the corollary follows from Theorem 4.4 and induction on $e$. Let $\mathfrak{p}=\mathfrak{P} \cap R$. By assumption either $E_{\mathfrak{p}}$ has a free summand of rank $e-1$ or $R_{\mathfrak{p}}$ is regular. It is clear that $R_{\mathfrak{P}}^{\prime \prime}$ is regular if $R_{\mathfrak{p}}$ is regular. Moreover, $R_{\mathfrak{p}}$ is regular if depth $R_{\mathfrak{p}} \leq 1$. Hence we may assume that depth $R_{\mathfrak{p}}=2$ and $E_{\mathfrak{p}}$ has a free summand of rank $e-1$. We write $E_{\mathfrak{p}}=R_{\mathfrak{p}} \oplus L$, where $L$ has a free summand of rank $e-2$. Now the argument of the previous proof shows that $\left(E^{\prime \prime} / R^{\prime \prime} x\right)_{\mathfrak{P}} \cong R_{\mathfrak{P}}^{\prime \prime} \otimes_{R_{\mathfrak{p}}} L$, which has a free summand of rank $e-2$.

Under suitable hypotheses, the previous theorem and corollary say that if a module is integrally closed then the specialization by a generic element of a reduction is integrally closed and so is the generic Bourbaki ideal with respect to a reduction. We finish by proving the converse in a very general setting. 
Proposition 4.6. Let $R$ be a Noetherian normal local ring and let $E$ be a finitely generated torsion-free $R$-module with rank $e \geq 2$. Let $U=\sum_{i=1}^{n} R a_{i}$ be a reduction of $E$. Let $R^{\prime \prime}=$ $R\left(z_{1}, \ldots, z_{n}\right), E^{\prime \prime}=R^{\prime \prime} \otimes_{R} E$, and $x=\sum_{i=1}^{n} z_{i} a_{i}$. The $R^{\prime \prime}$-module $E^{\prime \prime} / R^{\prime \prime} x$ is torsion-free, and if this module is integrally closed, then $E$ is integrally closed.

Proof. By Lemma 4.1, there exists an embedding $E \subset R^{e}$ such that $\nu\left(\left(R^{e} / E\right)_{\mathfrak{p}}\right) \leq e-1$ whenever depth $R_{\mathfrak{p}} \leq 1$. This embedding induces an embedding $U \subset R^{e}$ such that $\nu\left(\left(R^{e} / U\right)_{\mathfrak{p}}\right) \leq$ $e-1$ whenever depth $R_{\mathfrak{p}} \leq 1$ because $U_{\mathfrak{p}}=E_{\mathfrak{p}}$. It follows from Lemma 4.2 that $R^{\prime \prime e} / R^{\prime \prime} x$ is torsion-free of rank $e-1$. Hence there are embeddings $E^{\prime \prime} / R^{\prime \prime} x \subset R^{\prime \prime e} / R^{\prime \prime} x \subset R^{\prime \prime e-1}$, by which we obtain $E^{\prime \prime} / R^{\prime \prime} x \subset \overline{E^{\prime \prime}} / R^{\prime \prime} x \subset \overline{E^{\prime \prime} / R^{\prime \prime} x} \subset R^{\prime \prime e-1}$. Hence if $E^{\prime \prime} / R^{\prime \prime} x=\overline{E^{\prime \prime} / R^{\prime \prime} x}$, then $E^{\prime \prime}=\overline{E^{\prime \prime}}$ and therefore $E=\bar{E}$.

\section{REFERENCES}

[1] L. Avramov, Complete intersections and symmetric algebras, J. Algebra 73 (1981), 248-263.

[2] M. Brodmann and R. Sharp, Local cohomology: an algebraic introduction with geometric applications, Cambridge Studies in Advanced Mathematics 60, Cambridge University Press, 1998.

[3] W. Bruns and J. Herzog, Cohen-Macaulay rings, Cambridge Studies in Advanced Mathematics 39, Cambridge University Press, 1993.

[4] D. Buchsbaum and D. Eisenbud, Some structure theorems for finite free resolutions, Adv. Math. 12 (1974), 84-139.

[5] D. Eisenbud, C. Huneke, and B. Ulrich, What is the Rees algebra of a module?, Proc. Amer. Math. Soc. 131 (2003), 701-708.

[6] T. Gaffney, Polar methods, invariants of pairs of modules and equisingularity, Contemporary Math. 354 (2004), 113-135.

[7] T. Gaffney and S. Kleiman, Specialization of integral dependence for modules, Invent. Math. 137 (1999), $541-574$.

[8] R. Hartshorne, Complete intersections and connectedness, Amer. J. Math. 84 (1962), 497-508.

[9] M. Hochster, Properties of Noetherian rings stable under general grade reduction, Arch. Math. 24 (1973), 393-396.

[10] J. Hong, B. Ulrich, and W.V. Vasconcelos, Normalization of modules, J. Algebra 303 (2006), 133-145.

[11] C. Huneke, Hilbert functions and symbolic powers, Michigan Math. J. 34 (1987), 293-318.

[12] C. Huneke, Complete ideals in two-dimensional regular local rings, Commutative algebra (Berkeley, CA, 1987), Math. Sci. Res. Inst. Publ. 15, 1989, pp. 325-338.

[13] C. Huneke and M.E. Rossi, The dimension and components of symmetric algebras, J. Algebra 98 (1986), $200-210$.

[14] C. Huneke and J. Sally, Birational extensions in dimension two and integrally closed ideals, J. Algebra 115 (1988), 481-500.

[15] S. Itoh, Integral closures of ideals generated by regular sequences, J. Algebra 117 (1988), 390-401.

[16] S. Itoh, Coefficients of normal Hilbert polynomials, J. Algebra 150 (1992), 101-117.

[17] D. Katz and V. Kodiyalam, Symmetric powers of complete modules over a two-dimensional regular local ring, Trans. Amer. Math. Soc. 349 (1997), 747-762.

[18] V. Kodiyalam, Integrally closed modules over two-dimensional regular local rings, Trans. Amer. Math. Soc. 347 (1995), 3551-3573.

[19] J. Lipman and B. Teissier, Pseudo-rational local rings and a theorem of Briançon-Skoda about integral closures of ideals, Michigan Math. J. 28 (1981), 97-116.

[20] M. Miyazaki and Y. Yoshino, On heights and grades of determinantal ideals, J. Algebra 235 (2001), $783-804$.

[21] D. Rees, A note on analytically unramified local rings, J. London Math. Soc. 36 (1961), 24-28.

[22] D. Rees, Reduction of modules, Math. Proc. Camb. Phil. Soc. 101 (1987), 431-449.

[23] A. Simis, B. Ulrich, and W.V. Vasconcelos, Rees algebras of modules, Proc. London Math. Soc. 87 (2003), 610-646.

[24] I. Swanson and C. Huneke, Integral closure of ideals, rings, and modules, London Mathematical Society Lecture Note Series 336, Cambridge University Press, 2006. 
[25] B. Teissier, Cycles évanescents, sections planes et conditions de Whitney, Singularités à Cargèse, Astérisque 7-8, 1973, pp. 285-362.

[26] B. Teissier, Résolution simultanée et cycles évanescents, Séminaire sur les singularités des surfaces (Proc. 1976-1977), Springer Lect. Notes Math. 777, 1980, pp. 82-146.

[27] O. Zariski and P. Samuel, Commutative algebra, vol. II, Graduate Texts in Mathematics 29, SpringerVerlag, New York-Heidelberg, 1975.

Department of Mathematics, Southern Connecticut State University, 501 Crescent Street, New Haven, CT 06515

E-mail address: hongj2@southernct.edu

Department of Mathematics, Purdue University, 150 N. University Street, West Lafayette, IN 47907

E-mail address: ulrich@math.purdue.edu 